1 The Iowa Test of Consonant Perception

\title{
Validation of the Iowa Test of Consonant Perception
}

5 Jason Geller ${ }^{1}$, Ann Holmes ${ }^{1}$, Adam Schwalje ${ }^{2}$, Joel I Berger ${ }^{3}$, Phillip E Gander ${ }^{3}$, Inyong Choi ${ }^{4}$,

6 Bob McMurray ${ }^{1}$

$7 \quad{ }^{1}$ Department of Psychological and Brain Sciences, University of Iowa, G60 Psychological and

8 Brain Sciences Building, Iowa City, IA 52242

$9{ }^{2}$ Department of Otolaryngology - Head and Neck Surgery, University of Iowa, 200 Hawkins

10 Drive, 21151 Pomerantz Family Pavilion, Iowa City, IA 52242

$11{ }^{3}$ Department of Neurosurgery, University of Iowa, 200 Hawkins Drive, 1800 John Pappajohn

12 Pavilion, Iowa City, IA 52242

$13{ }^{4}$ Department of Communication Sciences and Disorders, University of Iowa, Wendell Johnson

14 Speech and Hearing Center, Iowa City, Iowa 52242

\section{Corresponding Author}

17 Jason Geller

18 Center for Cognitive Science

19152 Frelinghuysen Road

20 Busch Campus

21 Piscataway, New Jersey 08854

22 jason.geller@ruccs.rutgers.edu 


\section{Abstract}

26 Speech perception (especially in background noise) is a critical problem for hearing-impaired

27 listeners, and an important issue for cognitive hearing science. Despite a plethora of standardized 28 measures, few single-word closed-set tests uniformly sample the most frequently used phonemes

29 and use response choices that equally sample phonetic features like place and voicing. The Iowa

30 Test of Consonant Perception (ITCP) attempts to solve this. It is a proportionally balanced

31 phonemic word recognition task designed to assess perception of the initial consonant of

32 monosyllabic consonant-vowel-consonant (CVC) words. The ITCP consists of 120 sampled

33 CVC words. Words were recorded from four different talkers (two female) and uniformly sample

34 from all four quadrants of the vowel space to control for coarticulation. Response choices on

35 each trial are balanced to equate difficulty and sample a single phonetic feature. This study

36 evaluated the psychometric properties of ITCP by examining reliability (test-retest) and validity

37 in a sample of online normal hearing participants. Ninety-eight participants completed two

38 sessions of the ITCP along with standardized tests of words and sentence in noise (CNC words

39 and AzBio sentences). The ITCP showed good test-retest reliability and convergent validity with

40 two popular tests presented in noise. All the materials to use the ITCP, or to construct your own

41 version of the ITCP are freely available here: https://osf.io/hycdu/.

43 Keywords: auditory perception, speech-in-noise, closed-set, open-set, validation 


\section{Introduction}

Speech perception is generally successful under ideal circumstances, even as the auditory signal can be acoustically variable due to differences in talker speaking rate and coarticulation.

49 However, listening often takes place under non-ideal circumstances. For instance, background noise from other people and from machines is common, electronic communication can introduce

51 distortions or discontinuities in the overall message, and hearing loss is highly prevalent. Thus,

52 the way people cope with such circumstances is crucial for clinical work and basic science.

In the clinic, the most common challenge reported by hearing-impaired listeners is

54 understanding speech in noise (Hughes et al., 2018). Moreover, from a scientific perspective,

55 speech in noise perception may reveal a host of cognitive and cortical processes that are relevant

56 for speech but cannot be seen otherwise such as grouping features of the acoustic signal that

57 comprise the target speech, suppressing competing background elements, sustaining attention to 58 the target, and filling in missing information (Shinn-Cunningham, in press).

While the basic science of speech perception has often relied on stimuli that were

60 designed to test specific theories of perceptual organization or processing (e.g., Delattre et al.,

61 1955; Ganong, 1980; Remez et al., 1981), there is an equally important role for standardized

62 measures that assess listeners' relative skill at perceiving speech using representative speech

63 materials. This is fundamental for clinical work, but it is increasingly important in areas like

64 cognitive neuroscience. Here, such measures allow researchers to use stimuli that are

65 standardized and representative of speech/speech-in-noise $(\mathrm{SiN})$ in general. This can make it

66 easier to draw inferences across studies that use the same standard tests.

67 There is a wealth of such tests and materials available (e.g., California Consonant Test

68 [CCT], Owens \& Schubert, 1977; the Diagnostic Rhyme Test, Voiers, 1977, 1983); the Hearing 
69 in Noise Test [HINT], Nilsson, Soli, and Sullivan, 1994; the Modified Rhyme Test, House et al.,

70 1965; Words in Noise [WIN], Wilson, 2003; and the QuickSIN, Killion et al., 2004). Speech-in-

71 noise tests can be classified along two broad dimensions: 1) whether stimuli are sentences or

72 words, and 2) whether the response is open- or closed-set. There is no single "best" option for all

73 applications; however, these choices have implications for the inferences that can be made both

74 scientifically and clinically. Within the clinic and the lab, there is a strong zeitgeist toward

75 sentence-based, open-set tests, which are seen as more ecological. However, there is nonetheless

76 a clear need for better single-word, closed-set tests which we argue can address questions that

77 cannot be answered otherwise. Thus, this paper describes and validates the newly developed

78 Iowa Test of Consonant Perception (ITCP). We start by discussing these two core dimensions

79 (i.e., sentences vs. words and open-set vs. closed-set). We then describe the motivation, logic,

80 and development of the ITCP before presenting a validation study.

\section{A. Sentences vs. words}

A major difference between sentence- and word-based tests is demand characteristics.

83 The former requires correctly perceiving and repeating entire sentences (or the words within a

84 sentence), while the latter requires identifying (usually repeating out loud) a single word.

Sentence-based tests are generally seen as more predictive of outcomes for people with

86 hearing impairment. Sentence-based tests are arguably more ecologically valid and more

87 representative of real-world listening (e.g., human conversation). Because of this, for clinical

88 purposes at least, they are increasingly preferred over single word-based tests (Taylor, 2003).

Despite these arguments, sentence-based tests have two issues that may limit their use in

90 certain circumstances. First, at a purely auditory level, the phonemes of English (or any other

91 language) span many frequency ranges (Nakajima et al., 2017) and many types of acoustic cues 
92 (rapidly changing vs. steady-state, harmonic vs. aperiodic, etc.). These, in turn, are shaped by

93 neighboring sounds through coarticulation. Given the constraints on constructing reasonable

94 sentences, it may be difficult to sample this space uniformly, and some classes of sounds or some

95 coarticulatory contexts may be over- or under-assessed. This prevents such measures from

96 offering a truly representative measure of one's skill at speech perception and prevents them

97 from providing fine-grained diagnostic information about specific perceptual weaknesses.

98 Second, performance on sentence-based tests depends on several cognitive factors

99 unrelated to hearing. Perhaps the most common factor is context (Conrad, 1974). For example, in

100 the sentence, "She got a loaf of bread from the...", a listener can likely figure out that the last

101 word is "bakery" without hearing it. This sentence has a highly predictable context. However,

102 even sentences that are not predicable from context, which capture more bottom-up auditory

103 processing, can contain a lot of additional information that constrains perception. For example,

104 something as simple as a determiner ("the" or "a") can limit the range of lexical choices from

105 about 42,000 (the number of words in the average lexicon: Brysebaert, et al., 2016) to maybe

$106 \quad 15,000$ (just nouns or adjectives).

107 Moreover, sentence comprehension is a complex process in its own right. Indeed, entire

108 fields of cognitive science are devoted to understanding sentence processing in quiet. Some sub-

109 mechanisms like segmentation and prosody are highly perceptually based and cannot be

110 measured with isolated words (Salverda et al., 2003; Dilley \& Pitt, 2010). However, other

111 processes are only indirectly related to auditory perception. These include language-specific

112 processes bundled together as "sentence processing" which include parsing syntax, assigning

113 thematic roles to words, and establishing the referents of nouns and pronouns (see Tanenhaus

114 and Trueswell, 1995; Altmann, 1998 for reviews). Even at the level of recognizing the individual 
115 words in a sentence, the time demands created by sentences may challenge the system further as

116 listeners must recognize words rapidly as subsequent words may be unfolding. Finally, domain-

117 general processes like working memory and cognitive control are correlated with sentence

118 processing (Just \& Carpenter, 1992; Novick et al., 2005). In fact, the ability to hear a sentence in

119 quiet and repeat it is so consistently impaired in Developmental Language Disorder (a disorder

120 for which a hearing impairment is disqualifying) that it is considered a strong diagnostic feature

121 (Redmond et al., 2019). Thus, as a basis for measuring speech perception, sentence materials tap

122 into far more than speech perception.

123 The fact that all these processes contribute is likely why sentence-based tests are

124 generally more predictive than isolated words - they capture more between-listener variance

125 (Mueller, 2001; Taylor, 2003; Walden et al., 1983). However, much of this variance is due to

126 factors outside the auditory periphery and even outside cognitive processes that are reasonably

127 considered auditory. These factors pose even more significant problems when using sentence-

128 based tests with individuals with more variation in cognitive ability, such as older individuals

129 (Craik \& Bialystok, 2006; Peelle \& Wingfield, 2016), children, or individuals with neurological

130 or developmental disorders. These groups may have substantially more variance due to cognitive

131 factors, and it may be challenging to isolate variance in performance to perceptual processes.

132 Additionally, there has been an increasing interest in examining difficult listening beyond

133 intelligibility, by assessing how noise demands additional effort (Ohlenforst et al., 2017;

134 Pichora-Fuller et al., 2016; Saramplis et a., 2009), or changes in the cortical network (Du et al.,

135 2014; Kim et al., 2020; Wong et al., 2008; Bidelman et al., 2016). Sentence materials can make it

136 difficult to isolate such differences to specifically auditory processes. For example, listeners may

137 exert greater effort (e.g., greater pupil size in a pupillometry study) when attending to SiN; 
138 however, it is not clear if listeners are allocating more effort to sentence processing, for example,

139 rather than to auditory processes like streaming, grouping, selective attention, or speech

140 perception.

141 Single-word tests - though currently somewhat less favored - may mitigate some of

142 those issues. In contrast to sentence-based tests, typical single-word tests require listeners to

143 repeat or choose single words. These are typically seen as less ecologically valid than sentence-

144 based tests because they remove many extraneous individual differences underlying sentence-

145 based tests. However, single-word tests afford some advantages.

146 First, because the selection of words is less constrained by plausibility factors, single

147 word tests can sample the space of possible phonemes and coarticulatory contexts deliberately.

148 As we describe shortly, the distribution of items could be chosen to match the frequency of those

149 kinds of items (e.g., phonemes) in the world (maximizing predictive validity), or it could be done

150 uniformly so that all phonemes are tested roughly equally (maximizing diagnostic strength). A

151 uniform set of items, in particular, can potentially allow the clinician or researcher to isolate

152 specific perceptual issues. For example, single-word tests can provide information about the

153 discrimination of phonetic features, prosodic characteristics, and timing aspects of speech signals

154 (Blarney et al., 1987; Geers \& Brenner, 1994; Tyler et al., 1984).

155 Moreover, single words lessen memory demands and much of the complex cognitive

156 operations associated with sentence processing, providing a potentially purer estimate of auditory

157 or perceptual processing. This is not to say that single word tests have no cognitive demands.

158 There are factors at the lexical (word) level that may impact single-word performance including

159 word frequency (Elliott, Clifton, \& Servi, 1983) and neighborhood density (e.g., Neighborhood

160 Activation Model; Luce, 1986; Luce \& Pisoni, 1998). The process of word recognition is a 
161 complex cognitive process (e.g., Dahan \& Magnuson, 2006 for a review). Nonetheless, all of

162 these effects are present in sentence tests in addition to memory and sentence processing

163 constraints. Thus, on balance, single word tests may do a better job isolating auditory from

164 cognitive processes, using acoustically complex, real-world stimuli. This potentially may reduce

165 the variability occurring exclusively as a result of non-auditory factors.

166 For research purposes, the use of single words allows more precise stimulus control and

167 measurement of specific auditory factors involved in SiN perception (e.g., classes of phonemes,

168 phonetic dimensions like voicing, consonants vs. vowels, etc.) as there is more freedom to select

169 a representative distribution of sounds, and the simpler stimuli may allow the researcher to

170 isolate the effect of a single contrast on performance. Critically, single word tests may also be

171 more appropriate for temporally sensitive tools like magnetoencephalography (MEG),

172 electroencephalography (EEG), pupillometry, and eye-tracking studies where fast-changing

173 neural and physiological measures are time-locked to the stimulus.

\section{B. Open vs. closed-set tests}

The use of single word tests opens a second dimension on which SiN tests vary. In a

176 typical testing scenario, clinicians and researchers can use either forced-choice (closed-set) or

177 open-ended (open-set) tests. In the closed-set format, the listener receives a restricted list of

178 words or letters to select from. One is correct; the other choices are foils. In an open-set format,

179 listeners repeat back (or type) each word with no constraints imposed by the task. Here, the

180 critical distinction is not so much the response modality (e.g., button-pushing vs. vocal) but

181 whether the choices are constrained (closed set) or not (open).

182 There are several advantages to open-set tests. First, open-set tests are more difficult and 183 avoid ceiling effects (even potentially at higher [easier] signal-to-noise ratios [SNRs]). Second, 
184 open-set tests are not influenced by the response set. For example, in a closed set task, if a

185 listener misheard a $/ \mathrm{b} /$ as an $/ \mathrm{m} /$, but the choices were $/ \mathrm{b} /, / \mathrm{d} /$, and $/ \mathrm{g} /$, then $/ \mathrm{b} /$ may still be the

186 closest match to their percept. As a result, they may choose the correct answer even though they

187 misheard the speech. In this case, there is no way to measure the mistake. Thus, the difficulty of

188 an item is shaped by the available choices. This can be problematic if response sets vary in

189 difficulty across words (e.g., if all the labials are tested in easier sets than the fricatives). This can

190 make it look like some sounds are more or less difficult, when the response sets are actually

191 driving this. Finally, the lack of constraint in open-set tests may allow them to tap a wider range

192 of factors that are known to influence speech processing such as talker variability and lexical

193 competition (e.g., Clopper et al., 2006).

194 Despite these advantages, the open-set response format is not necessarily more

195 ecologically valid than a closed- set task for three reasons. First, in everyday conversation,

196 listeners map speech to meaning - they rarely need to repeat back what they hear. However, the

197 neural pathways dedicated to mapping incoming speech to production are distinct from the

198 pathways dedicated to meaning (e.g., Hickok \& Poeppel, 2007). While open-set tests do not

199 always involve speech production per se (some involve typing), all open set tests require

200 listeners to map sound to its phonological, articulatory, or orthographic form. Consequently,

201 open-set tests may not tap the pathways commonly used.

202 Second, and relatedly, open set responding brings in speech production processes. Here,

203 speech production refers to a broader set of processes than just articulation, including selecting

204 and retrieving a word from the lexicon and generating a complex motor response. If the goal is a

205 pure assessment, this may just add variance and could even add to predictive validity. Like the

206 issues raised for sentences, some of this variance may be due to processes that have nothing to do 
207 with speech perception. Using a writing or typing task (instead of articulation) does not obviate

208 the problem. Emerging work suggests that typing is subject to many of the same linguistic

209 influences as word production and introduces more factors (sound/spelling regularity; Pinet et

210 al., 2016).

211 Finally, the interaction of the cognitive processes involved in speech production with

212 those involved in word recognition may impose more uncertainty on the measure. For example,

213 the presence of lexical neighbors (e.g., words that differ by one phoneme) interferes with

214 recognition (Luce, 1986; Luce \& Pisoni, 1998), but facilitates speech production (e.g., Gordon,

215 2002; Middleton \& Schwartz, 2010; Chen \& Mirman, 2012). It is unclear how these forces may

216 play out in special populations.

217 In the clinic, the fact that open-set tests rely on speech production may have other

218 consequences. Open-set tests are harder to administer to participants with speech production

219 deficits (e.g., Aphasia). They may also be problematic in children who can take 18 years to

220 develop adult-like speech production (Sadagopan \& Smith, 2008). In these groups, some open-

221 set performance variation could be related to speech production, not SiN processing. Moreover,

222 researchers are beginning to document that speech pathologists may struggle to identify

223 children's productions in some cases (c.f., Munson et al., 2012), raising the issue of assessor

224 reliability with individuals with disabilities or children.

225 The closed-set format overcomes some of these issues (even as it is subject to the

226 concerns described above) as it does not require speech production. Moreover, the closed-set

227 testing format is economical - tests can be rapidly administered and scored automatically

228 without a human coder (Black, 1957), which is more efficient and can enable adaptive testing.

229 Finally, by carefully choosing foils for each word, these assessments can pinpoint specific 
230 perceptual difficulties at the feature- or phoneme-levels (Foster \& Haggard, 1987). In the context

231 of cognitive science and cognitive neuroscience, closed-set tests are highly advantageous as they

232 minimize cognitive and cortical components due to speech production.

233 It is important to note here that closed-set tests do require a response (e.g., button presses

234 or typing responses) which may make it harder for individuals from special populations.

235 However, these responses (e.g., a button press or typing a response) are motorically simpler than 236 speech production, and importantly, they are not inherently linguistic. This is important because 237 individuals with a language or perception disorder are much more likely to face impairments in 238 speech production than in button pressing. Moreover, inherently linguistic responses may be 239 affected by many of the same linguistic factors that affect speech perception (e.g., neighborhood 240 density) but in different ways. By using a simpler, non-linguistic response, these issues can be 241 minimized (though not always avoided).

242 One common concern is that closed-set tests may not be sensitive to the same cognitive 243 demands involved in everyday listening situations (e.g., Clopper et al., 2006; Sommers et al., 244 1997). Indeed, Sommers et al. (1997) found that performance in a closed-set SiN task was not 245 affected by lexical competition (neighborhood density) or talker variability, whereas 246 performance on an open-set task was. However, Clopper et al. (2006) showed that closed-set 247 tests can better approximate speech perception by increasing response options (e.g., from 4 248 options to 6 options) and number of talkers. Thus, it is not clear whether these limits are because 249 people don't engage these broader cognitive processes in closed-set tests, or they do engage 250 these processes, but their influence is minimized by ceiling effects in some specific tests.

251 Even though single-word closed-set SiN tests are somewhat out of vogue, these tests have 252 some advantages in that they can isolate auditory processes from many higher-level cognitive 
253 processes (like sentence processing, speech production and short term memory); they can

254 uniformly sample the phonetic space, and potentially isolate specific contrasts (e.g., voicing);

255 they can be scored automatically and efficiently; and the timing properties can be controlled for

256 in domains where timing precision is needed (i.e., cognitive neuroscience). Thus, The ITCP

257 developed here uses the closed-set format.

258 One challenge in developing an ideal closed-set single-word task is identifying a set of

259 response options for each word that effectively samples the possible responses a listener may

260 consider but without biasing the subject. Some tests go so far as to use many possible responses

261 and hold this consistent across trials (Clopper et al., 2006). This requires one to use orthographic

262 (letter) stimuli - which may require a level of phonological awareness that can be problematic

263 for children or people with brain damage. It may also interact in odd ways with the choice of

264 stimuli. For example, if the target word was fall, the phonemes /v/ and / $\theta /$ can be ruled out as vall

265 and thall are not real words. Thus, while the use of all possible responses may appear to make

266 this more of an open-set task, functionally speaking, it is not, and the functional number of

267 reasonable foils may differ from word to word.

268 Alternatively, some closed-set tests may adopt a fixed number of foils and change the

269 response options on each trial (e.g., CCT, Owens \& Schubert, 1977). This approach allows the

270 use of whole words as response options, reducing meta-phonological demands (while still

271 requiring reading ability). When doing so, however, it may be challenging to control difficulty as

272 the set of foils can be arbitrarily close (e.g., /b/ vs. /p/), far (/b/ vs. / / /), or may unintentionally

273 stress certain phonological contrasts over others (e.g., if all the foils come from the same voicing

274 or manner class). The ITCP attempts to mitigate this by controlling the target / foil relationships

275 (e.g., the number of trials where there was a foil that contrasted by only one feature), by using a 
276 computer-based search algorithm to find a set of targets and foils that constrain the range of

277 difficulty, and with an IRT analysis to further refine this set.

278 C. Goals

279 With these issues in mind, our primary goal was to develop a single-word, closed-set task

280 that attempts to solve many of the aforementioned issues. Our immediate motivation was to

281 develop a task for cognitive neuroscience studies, where single-word tests allow for better

282 control of stimuli and their timing, eliminates cortical processes related to speech production,

283 and can better control the phonetic contrasts that are tested to ensure that we are roughly

284 sampling the entire acoustic/phonetic space. However, the development and validation of this

285 test was psychometrically oriented to enable this to be used across other studies and shared with

286 other labs, and potentially to develop into a measure of clinical utility.

287 This paper serves two purposes. First, we describe the development and structure of the

288 ITCP. Second, we present the results of our full validation experiment.

289 II. The Iowa Test of Consonant Perception (ITCP)

290 The ITCP was developed to improve upon typical closed-set SiN tests to provide a test of

291 assessing consonant perception in noise using real words. We sought to address four limitations

292 of typical single-word tests (both closed- and open-set) to create an assessment which as nearly

293 as possible uniformly samples the space of possible phonemes and contexts.

294 First, in typical tests, it is common for words to be produced by a single talker (c.f.,

295 Torretta, 1995). This is problematic for three reasons. First, the use of a single talker eliminates

296 one of the largest sources of acoustic variability present in everyday speech perception

297 (McMurray and Jongman, 2011; Newman et al., 2001; Cole et al., 2010), where interaction with

298 multiple talkers is typical (Clopper et al., 2006). Second, hearing loss and the use of 
amplification devices can make some talkers more intelligible than others. For example, in a bimodal cochlear implant (CI) configuration (a CI plus low-frequency acoustic hearing in the contralateral ear), a listener may perform well with male talkers since the pitch is in range of the acoustic ear, but struggle with female talkers whose pitch is in the electrical stimulation range. Finally, from a measurement perspective, talkers vary in their intelligibility due to things like

304 dialect, articulatory precision, and rate. With a single talker, one may not achieve a robust, 305 generalizable measure of speech perception. Therefore, the ITCP uses four talkers (two female). Second, single word tests allow the researcher to sample the acoustic phonetic space deliberately. In this regard, there are two philosophies reflecting distinct goals. One can strive for a representative sample by which the distribution of phonemes matches their frequency or utility in the world (e.g., the CNC words; Lehiste \& Peterson, 1959). This provides potentially

310 better predictive validity, but comes at the cost of diagnostic strength. Some phonemes may not

311 be sampled sufficiently to isolate the source of a listener's difficulty. Alternatively, one can 312 strive for uniformity by sampling all phonemes equally. This can help reveal the precise nature 313 of a hearing deficit but comes with a predictive validity cost. Both philosophies have utility, but 314 here we focused on uniformity, as we sought a test to help identify specific problems for both 315 clinical and basic research. Long term, future work should be able to develop weighted accuracy 316 measures (e.g., weight the frequency of each phoneme in the language) from this uniformly 317 sampled assessment (in the same way that the speech intelligibility index is computed from a 318 uniformly sampled audiogram). In contrast, converting a score from a representative (frequency 319 weighted) measure to a uniform one may not be possible. Many existing single-word measures do not attempt to sample the space of consonants

321 and vowel spaces uniformly. For illustrative purposes, Figure 1 shows the distributions of onset 
322 consonants tested (Figure 1) in two common tests, the CNC (an open-set task) and the CCT test

323 (a closed-set task), along with similar measures for the ITCP. In these plots, the width of the arc

324 illustrates the number of trials testing a specific onset phoneme. Open set tests such as the CNC

325 can be scored based on all phonemes, and the CCT has foils to test both onset and coda

326 consonants. But for comparison to the ITCP — which is designed to test primarily onset

327 consonants - only onset consonants are considered for the CNC and CCT distributions. As

328 Figure 1 shows, no single task is fully balanced: each misses several phonemes, but many are

329 oversampled. The CNC test, for example, oversamples $/ \mathrm{w} /$ and under-samples $/ \mathrm{g} /, / \mathrm{n} /$ and the

330 non-sibilant fricatives; whereas the CCT dramatically under-samples /g/ and /d/ and misses

331 several of the fricatives and sonorants altogether. This is not meant to critique these tests

332 individually but to illustrate the differences in phoneme sampling.

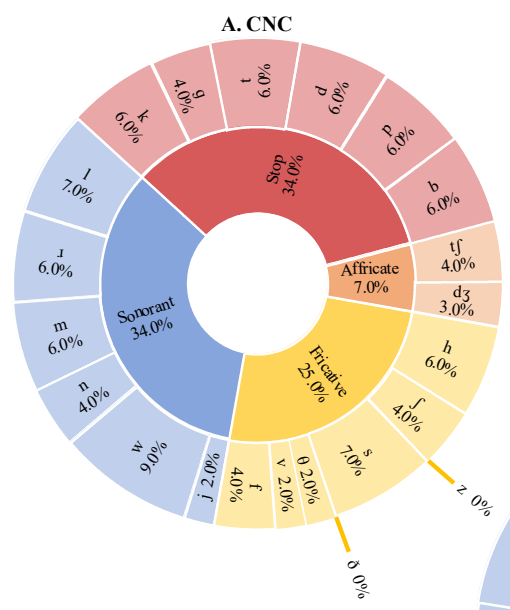

Figure 1. (Color online). Distribution of consonants tested in two common tests and the ITCP (A. CNC; B. CCT; C. ITCP). Consonants are color coded by manner of articulation, and the width of each arc corresponds to the number of trials. 
There are numerous reasons for this lack of a uniform distribution. There may be insufficient items in the language, or the test may focus only on phonemes that are the most

337 frequently used. Consequently, results may not generalize to all English sounds; instead, such

338 tests may capture largely stop consonant perception (for example). Thus, the ITCP samples most

339 of the onset consonants of English and does so with an approximately equal number of trials for

340 each phoneme and/or classes of phonemes (e.g., stops, fricatives, etc.; see Figure 1C).

342 available response options influence performance. For example, even if a listener cannot hear the

343 difference between /b/ and /d/, they could still distinguish beak, teak, peak, and leak (since /d/ is

344 not an option). Similarly, a test that always contrasted $/ \mathrm{b} /$ and $/ \mathrm{p} /($ but never $/ \mathrm{b} /$ and $/ \mathrm{d} /$ ) is

345 functionally a test of voicing discrimination, not speech perception writ large. Thus, it was

346 crucial not only that the target sounds in the ITCP span the full phonetic space but also that the

347 contrasts established by each set span the phonetic space as well.

348 To accomplish this, the ITCP uses four response choices on each trial (the target + three

349 foils), which balances phonetic contrasts across items to the extent that an English word is

350 available to serve as a foil. Heat maps in Figure 2 show the frequency (count) of target + foil

351 contrasts for the ITCP ${ }^{1}$. Four response options were chosen because (1) it allows each trial to tap

352 multiple contrasts; and (2) it is difficult enough to avoid ceiling effects; and it is small enough to

353 avoid overloading working memory. All four options are minimal pairs that only differ on the

354 initial consonant, and at least one

\footnotetext{
${ }^{1}$ Not all contrasts are possible in English. It is not possible to contrast sonorants in voicing (there is no voiceless counterpart to $/ 1 /$ and $/ \mathrm{m} /$ for example) and $/ \mathrm{J} /, / \theta /$, and $/ ð /$, have virtually no minimal pairs for voicing. There are also no manner contrasts for $/ \mathrm{k} /, / \mathrm{g} /, / \theta /$ and $/ ð /$ as those places of articulation only appear in one manner class. This is highlighted in Figure 2 with red boxes
} 


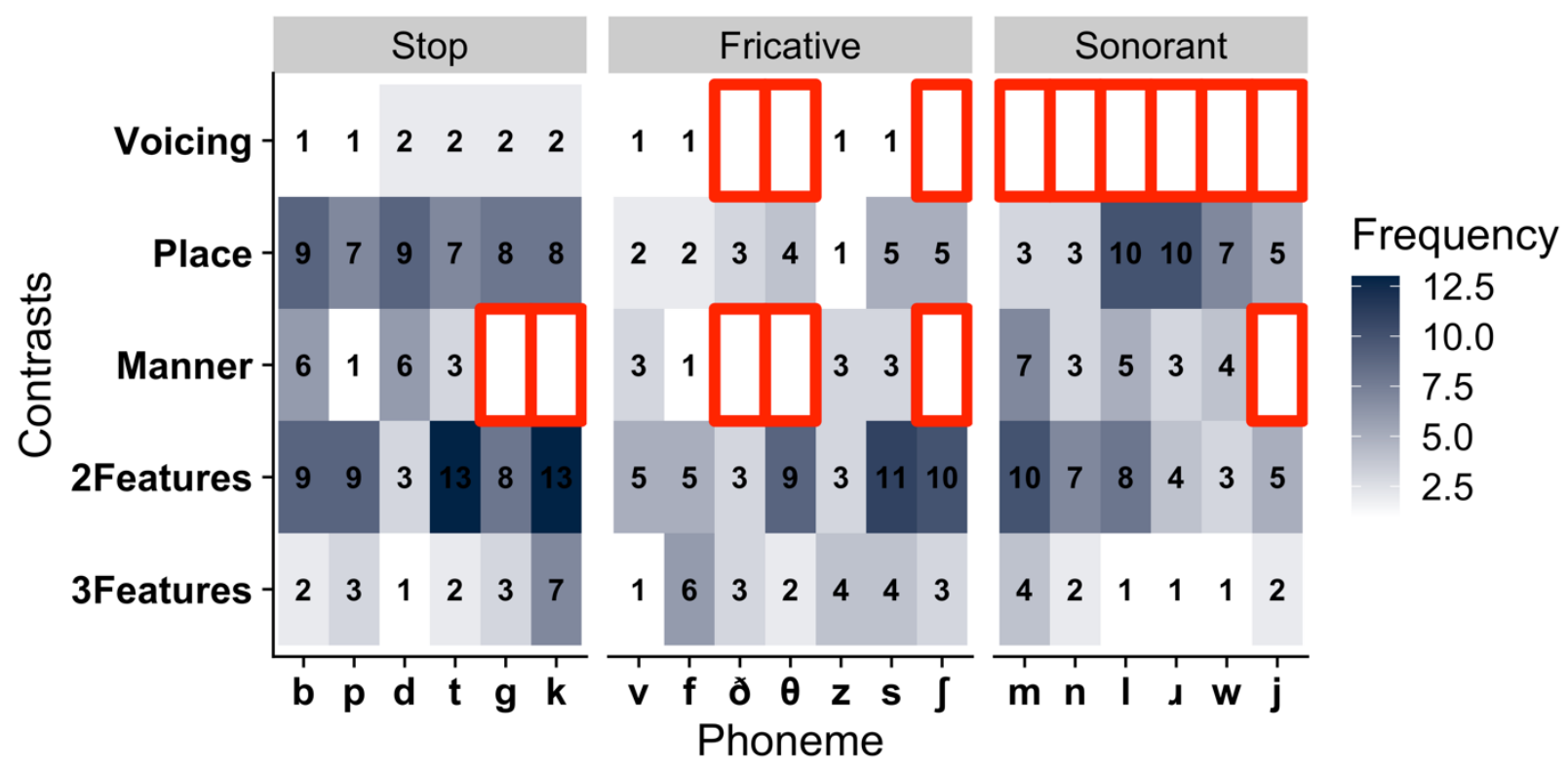

356 (if not more) differ only on one phonetic feature (place, manner or voicing). Critically, the

357 specific foils chosen for each word were based on a massive computational search through the

Figure 2. (Color online). Heat map showing the number of trials that test a specific minimal pair contrast for each phoneme. For example, $\mathrm{a} / \mathrm{b} /$ trial testing place would use either a $/ \mathrm{g} / \mathrm{or} / \mathrm{d} / \mathrm{as}$ a foil; $\mathrm{a} / \mathrm{z} / \mathrm{trial}$ testing voicing would have $/ \mathrm{s} /$ for a foil, and a $/ \mathrm{z} /$ trial testing manner would have $/ \mathrm{n} /, / \mathrm{d} /, / 1 /$ and $/ \mathrm{d} /$ for foils. Note that some contrasts (indicated in white with red boxes) are not possible in word-initial positions in English (sonorants cannot contrast voicing, and some places of articulation are found only in fricatives) or were so rare that no minimal pairs could be formed. Also note that we did not attempt to collapse neighboring places of articulation that are commonly treated similarly (e.g., /k/ [velar] and / $\mathrm{f} /$ [postalveolar]) are treated as a 2-feature contrast [place + manner]; thus many two-feature changes are actually quite close in acoustic or articulatory space.

358 space of English words (Schwalje, Choi \& McMurray, in prep; described shortly) to identify a

359 set of items in which the foils — as much as possible - uniformly sampled the space of possible

360 contrasts, and are as close as possible to expressing a single feature (place, voicing, and manner)

361 contrast. This automated procedure was intended to reduce variation in difficulty across items

362 that were due to the specific foil choices.

363 Finally, few speech perception tests systematically consider the role of coarticulation -

364 the degree to which neighboring sounds influence each other. This is widely seen as a core

365 source of acoustic ambiguity for normal hearing listeners as a /b/, for example, can be 
366 dramatically different depending on the neighboring vowels (Delattre et al., 1955; McMurray \&

367 Jongman, 2011). Ignoring coarticulation may create two problems. First, the ability to

368 compensate for coarticulation may be fundamental for good speech perception (McMurray \&

369 Jongman, 2011). Second, coarticulation can shape the frequency profile in ways that could be

370 confounded with performance. Lip rounding (from an $/ \mathrm{u} /$, for example) lowers the preceding

371 consonant spectrum (Daniloff \& Moll, 1968). If /g/, for example, is only tested in a /u/ context

372 but a /d/ is tested in an /i/ context, a listener who struggles with low-frequency sounds (e.g., a

373 standard CI user) may appear to perform worse on velars than coronals when the difference in

374 coarticulatory contexts is actually driving this. Thus, it is essential to test sounds in a range of

375 contexts. However, as Figure 3 shows, this is not commonly considered by existing tests (panels

376 A and B) and consequently many vowel contexts are dramatically under or over sampled.

377 The ITCP overcomes this by testing each consonant with four vowels that roughly span

378 the quadrants of the vowel space, where maximal coarticulation would be exerted (see Figure 3

379 for the distribution of trials as a function of vowel context and Figure 4 for the co-occurrence of

380 consonants with each of four vowel types). This was motivated by our goal of uniformity

381 (permitting better diagnosticity) rather than representativeness (some CV pairs are more likely

382 than others). Non-corner vowels were excluded to minimize the number of trials and because we

383 could adequately sample the dimensions of frontness, and height from the corners.

384 Finally, we constrained the items in the ITCP to be real English words. This was done to 385 ensure applicability to older adults, children, and people with brain damage (many of which are 386 elderly) who may struggle with non-words, and to avoid lexical biases if a mix of words and 387 nonwords were used (c.f., Coady \& Evans, 2008; Glosser et al., 1997; Rogalsky, 


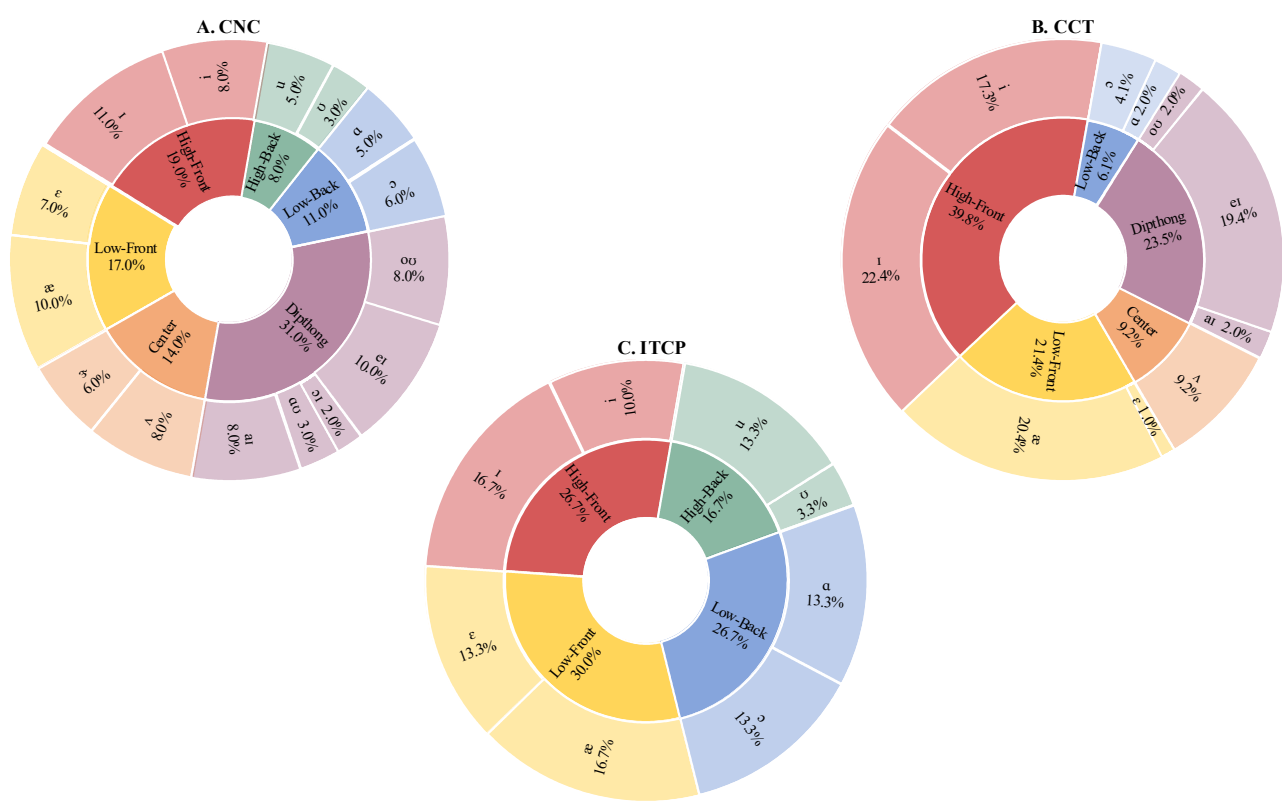

389 Figure 3. (Color online). Distribution of vowels by type in two common speech tests and the ITCP (A. 390 CNC; B. CCT; C. ITCP). Vowels are color coded by manner of articulation, and the width of each arc 391 corresponds to the number of trials. 2015). As a result of this constraint, and the constraint that all four words in a set needed to be

394 close minimal pairs, there were surprisingly few words available, and there were simply not 


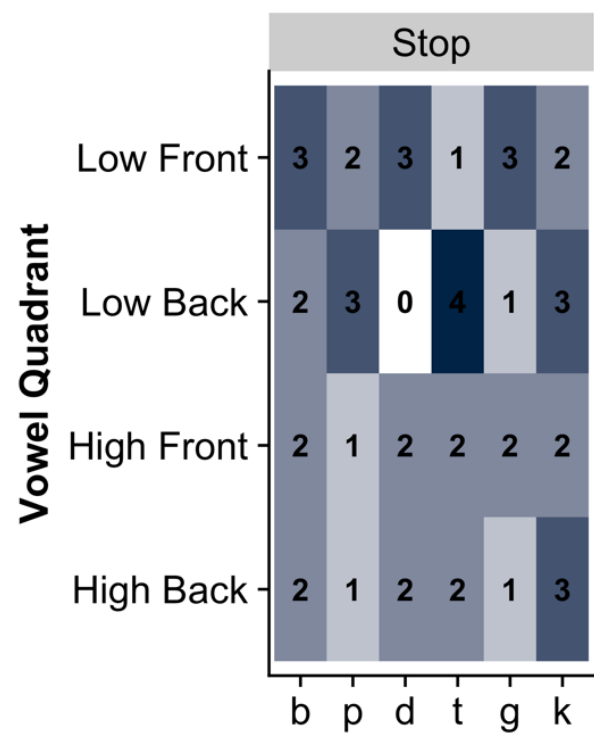

398 repeat trials to make sure of this). Third, no word appears in multiple sets. Fourth, to mitigate the
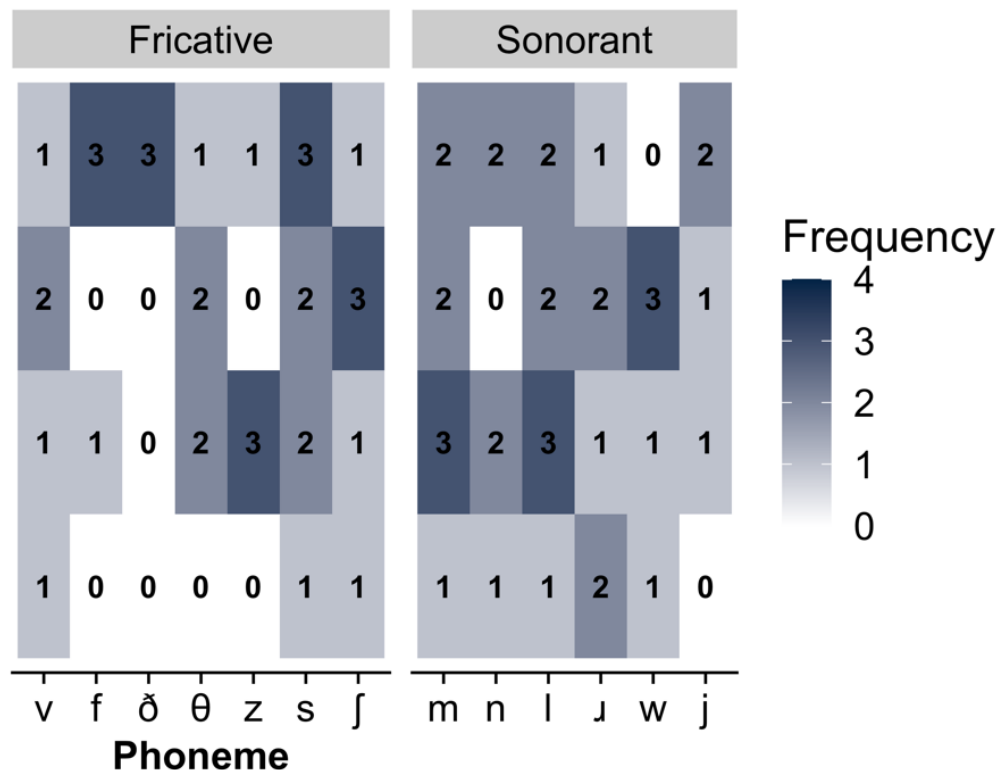

Figure 4. (Color online). Consonant by vowel-type cooccurrence counts for the 120 items in the ITCP. Color intensity represents magnitude with lighter colors representing a fewer number of trials testing a particular contrast within a phoneme and darker colors representing a greater number of trials testing a particular contrast within a phoneme.

enough words in English to use a given set only once (since a set would use up four words - the target and three foils, which would never even be heard).

Instead, the ITCP cycles through each of the four words in a set, and sets can then be used multiple times with a different target word. While repetition is not generally a good design feature, we took several steps to minimize learning effects. First, by cycling through each of the four items in a set, all words are equally likely. Thus, seeing the set van/than/fan/man does not prime the listener to expect a specific target word. Second, cycling through a whole set does raise the possibility of a "process of elimination" strategy. For example, after a van trial and a fan trial, subjects can rule those two words out on subsequent trials with that set. To avoid this, each word is heard at least twice (in some cases, the researcher might want to add several randomly selected potential for participants to memorize the "exemplars" (the specific idiosyncratic acoustic 
411 properties of a given recording), each word is only heard once by a given talker. Fifth, the task is

412 fully randomized, which prevents participants from building up expectations about words they

413 have heard or not heard. Finally, each item in a set of four is heard an equal number of times

414 (excluding any repeat trials that were added). This lessens the influence of frequency and

415 neighborhood effects by counterbalancing item-specific factors (e.g., than is more frequent than

416 van in English, thus subjects may be more accurate for this item; however, by also testing them

417 on their perception of van and averaging the results we can factor out frequency).

418 The ITCP tried to simultaneously satisfy all these constraints: the use of real words,

419 relative uniform testing of each consonant, relatively balanced difficulty expressed in the foils,

420 and an even distribution of coarticulatory contests. However, given the constraints of English, it

421 could not be perfect. Sometimes, there were insufficient words available to ensure minimal close

422 pairs, or the constraints of testing in sets of four items disrupted uniformity. For example, a set

423 like ban, man, van, and than tests several manner-of articulation contrasts, for places of

424 articulation near the front of the mouth (labials, labiodentals and dentals). However, it is missing

425 the sonorant, /w/. To test /w/, we would have to add another set (wall, ball, fall, and shawl), but

426 that adds an extra labial, sacrificing uniform sampling of the consonants, and it adds a less-close

427 contrast (e.g., /w/vs. / / /). Thus, the ITCP (and likely any similar test) cannot perfectly live up to

428 the goals here. To maximize our satisfaction of these constraints, we exhaustively searched a

429 million possible sets to find a reasonable set of sets. We then used an item-response-theory

430 approach to constrain items further.

431 All raw and summary data, analysis code, and materials related to the ITCP are available

432 on our OSF website (https://osf.io/hycdu/). In this repository, we provide a list of the words with

433 the corresponding response options; sound files for the words recorded by four talkers in silence 
434 and noise; a MATLAB script that can be used to add multi-talker babble at a specified level; and 435 item difficulty estimates for each word which were measured in the study described below. We

436 note that the item database is coarsely coded by broad phonological categories (place, manner

437 and voicing), and in many cases we took liberties to avoid an overly fine-grained scheme that

438 would make it harder to generalize across sets. We do not intend this as a rigorous analysis of

439 the phonology of these words and it is provided for convenience of the users; more detail is

440 available in the "Design_Read_Me.docx" located here: https://osf.io/7cf6k/.

441 Our goal with the repository is that a user should be able to build smaller or larger

442 versions of the ITCP with any form of noise they want. In the repository, we also provide a

443 document describing the design principles of the ITCP to ensure that the resulting design is

444 appropriately balanced, and a table of the specific designs implemented here. We do not provide

445 a specific testing script. The one used here was developed in the online platform, Gorilla (and

446 can be viewed online at https://gorilla.sc/openmaterials/97811). This task should be simple

447 enough to implement in any existing experimental control platform. Those wishing to stay within

448 the Gorilla platform can simply clone our ITCP task using the link above.

\section{A. ITCP Development and Construction}

To create the ITCP, we used an iterative design process. First, we identified a large array

451 of possible sets (words plus foils) that could be pared down into a list of sets that met the design

452 principles (nearly uniform sampling, equal sampling across vowels, balanced difficulty based on

453 the foils). Second, we recorded all words, and conducted a pilot study in the lab. Third, following

454 pilot work, an Item Response Theory (IRT) analysis estimated the easiness of each item (word)

455 in order to identify outliers. Finally, we constructed the final set of items, discarding items that

456 were outliers in terms of easiness, but keeping phonetic balance in mind (e.g., a uniform 
457 distribution of phonemes and an equal balance of contrasts in the foils).

\section{Item development}

Item sets used monosyllabic words that ended in a consonant or cluster (CVC, and in a

460 few cases, CVCC words). Closed syllables (rather than CVs) were used because the final

461 consonant allowed additional vowels (e.g., /I/ and /æ/ are not permissible in an open syllable),

462 giving more available words. We then used an automated procedure to sift through about a

463 million possible sets of items, seeking sets that balanced the constraints outlined above.

464 We started by developing a comprehensive list of candidate words by pooling items from

465 the Clearpond (Marian et al., 2012), MRC (Wilson, 1988), and IPHOD (Vaden et al., 2010)

466 corpora. We used words with a frequency greater than 0.5 per $1,000,000$ according to the

467 SUBTLEX-US database (Brysbaert \& New, 2009) and words with high neighborhood density-

468 we discarded words with fewer than three near neighbors, as they could not form a set. At this

469 stage, any word that appeared in the corpora meeting the prespecified constraints was included.

470 These were hand-checked to ensure uniform phonemic transcriptions (across the corpora) and to

471 cull any words that were not reasonably well known. This process led to a master set of

472 candidates that was independent of the specific corpora.

473 Next, we constructed a series of sets of four words. Each set contained a target and three

474 foils (though the foils would also serve as targets on other trials). Sets were chosen pseudo-

475 randomly (using criteria described below) and were chosen without replacement so that words

476 could not appear in more than one set. The algorithm used to choose sets attempted to respect

477 the following goals, in order of priority: 1) The set of sets should comprehensively sample the

478 possible initial consonants (and be roughly uniformly distributed). 2) Each consonant should

479 appear with vowels from four broad classes (frontness $\times$ height). For this factor, we attempted to 
sample from all four quadrants of the vowel-space, but collapsed nearby vowels (e.g., /i/ and /I/ were both treated as high-front). 3) Sets should maximize within-set phoneme confusion (e.g., include as many single-feature contrasts as possible, e.g., /t/ vs. /d/, but not /t/ vs. /m/).

For each set of sets, this was done until we ran out of words to create the set of sets.

484 Finally, we evaluated the goodness of fit of each set of sets, balancing the three goals listed

485 above. We created one million sets of sets in this fashion. The resulting versions were compared and, for pilot testing, the three best-fit arrays with the least overlap between their member sets

487 were combined to form a candidate array of 48 sets comprising 191 words $^{2}$. We then piloted

488 those 191 words with listeners across two sessions to determine the difficulty of each set, to 489 further reduce the array size to the minimal sets needed for an adequate test (see Section 2.2).

\section{Auditory materials}

491 All words were recorded in Audacity (Audacity Team, 2012) using a Kay CSL 4300B

492 analog to digital board with a Shure-WH30 microphone in a sound-attenuated room. All words

493 were sampled at $44.1 \mathrm{kHz}$ and 16-bit depth. Four speakers of midland American English (two

494 male and two females ranging in age from 18-25 years) were instructed to speak all 191 words 2-

4953 times as clear as possible with and without the carrier phrase of "he said [word]". The carrier

496 phrase was used to help ensure uniform prosody and rate.

497 Raw recordings were first processed using Audacity's Noise Reduction algorithm which

498 estimates the spectrum of the ambient room noise and removes it from the entire raw sound file.

499 A check was performed over high-quality headphones to ensure that Audacity's Noise Reduction

500 algorithm removed excess room noise without introducing distortions. All exemplars of the same

\footnotetext{
${ }^{2}$ At this stage, the same word might appear in two sets; this was done to give more flexibility in choosing the final sets, and the repetition was eliminated after piloting.
} 
501 word (4-6 words per recording) were grouped together into a new WAV file, and then one token

502 of each word was selected as a final version of the word. Exemplars were selected to have

503 uniform prosody and duration, clear articulation, and be free of any artifacts (e.g., jaw clicks,

504 etc.). Each word was cut at zero-crossings in the WAV file as close to the start and end of the

505 word to isolate just the energy related to the single word. We then performed minor editing to

506 remove any remaining jaw clicks, loud aspiration, and other artifacts.

507 Finally, the senior author reviewed each selected token to determine if the token was a

508 good exemplar of that word, if another token should be selected, or if the word should be

509 rerecorded altogether. Talkers came back for at least one additional session to record any words

510 flagged for rerecording to follow the same methods listed above.

$511 \quad$ 3. Noise

512 Target and multi-speaker babble were digitally mixed using a MATLAB script (see our

513 OSF page) to create a series of WAV files with a dB SNR of -1.5 . For each trial, an isolated 1.5

514 second-long multi-talker babble noise was played. Babble-noise excerpts were extracted from an

515 original five-minute-long eight-talker babble noise from the Revised SPIN Test (a compact disc

516 published by Auditec, Inc., St. Louis, Missouri). All excerpts had a relatively constant RMS level

517 (within $+/-1 \mathrm{~dB}$ ), and a distinct excerpt was used for each word. There was no ramp-up or ramp-

518 down used. Target word onsets were at a random position between 0.5 and $1.0 \mathrm{~s}$, and noise

519 continued for a fixed period of 1.5 seconds.

520 The ITCP OSF site contains the MATLAB script for adding noise. The script is fully

521 customizable and can add any amount of noise with any temporal properties (e.g., the ramp, the

522 duration before the target, etc.). The user should be aware that a specific SNR in dB is difficult

523 to achieve automatically with real speech, and the dB SNR parameter is an estimate. True dB 
524 SNR should be measured in the sound field where the test will be administered.

525

526

527

528

529

530

\section{Loudness normalization}

To limit variation in loudness, especially for controlling signal-to-noise ratio, all words were amplitude-normalized. Automatic loudness normalization is difficult for two reasons. First, perceived loudness is a function of frequency (Dawson et al., 2017) and phonemes necessarily vary in spectral composition (e.g., a sibilant fricative will have more high frequency energy than a nasal). Second, it can be hard to quantify absolute loudness with a time varying envelope. Thus, we adopted a hybrid procedure implemented with custom MATLAB scripts using the Audio Toolbox (R2018b, MathWorks, MA, USA). As is typical, this starts with a single estimate of loudness for each file. Then the point-wise amplitude of each waveform is scaled by this estimate. For this single estimate, we started by identifying all the time points over which the amplitude was greater than $90 \%$ of the maximum for that word and used the RMS amplitude as the loudness. In practice, this meant scaling for each word was generally performed according to the amplitude of the vowel (according to the sonority hierarchy; Clements, 1990), the point that is most stable across words. This constraint ensured that quieter sections of the word (i.e., the closure between the vowel and the final consonant, a low amplitude obstruent) do not contribute to the loudness estimate used for normalization. We then scaled the amplitude of every point according to this value to achieve a uniform scaling in each sample. All WAV files were normalized in this way across talkers. Finally, after stimuli were normalized in this way, each token was reviewed by two members of the team (native English speakers) using high-quality headphones to identify individual words which were slightly louder or softer than the others within a talker. These had their total intensity increased or decreased by up to $10 \%$.

\section{B. Pilot Experiment 1}


The 48 sets identified in our initial procedure were tested in a pilot experiment. Normal-

548 hearing listeners $(N=30)$ heard each stimulus once through headphones. On each trial, individuals

549 saw a fixation cross for $500 \mathrm{~ms}$ and then listened to a word spoken by a single speaker in multi-

550 speaker babble (-1.5 dB SNR), at a random interval between $500 \mathrm{~ms}$ and $1 \mathrm{~s}$ from the onset. The

551 entire duration of the babble was $1.5 \mathrm{~s}$. Immediately after the offset of the babble, there was $1 \mathrm{~s}$

552 of silence. Following this, participants selected the word they heard amongst the four alternatives

553 specified by our procedure (see Figure 5 for a schematic).

554 Results were analyzed with a 1-parameter logistic (1-PL) random effects IRT model

555 using the glmer package in R (Bates et al., 2015) ${ }^{3}$. The IRT was used to identify outlier items

556 (i.e., items that were easier or harder than expected). This 1-PL model included random

557 intercepts of participant and item, and we used the estimated item intercept for each word as a 558 measure of its easiness/difficulty. The model also included fixed effects of 1) talker; 2) place,

559 voicing, and manner of the consonants; 3) vowel context; and 4) a limited set of interaction terms

560 (these were coded similarly to the analysis employed here in the validation study and described

561 in Section 4.3). The fixed effects tried to capture much of the known variance across phonemes

562 (e.g., that non-sibilant fricatives are generally more difficult than sibilants). As a result, each

563 word's estimated item intercepts can be interpreted as the difficulty or easiness of an item given

564 its phoneme class, vowel, and talker. An intercept of 0 means that the item is no harder or easier

565 than other similar items, where an intercept greater than 0 means it is easier than expected. This

566 fixed + random effects scheme was used because we knew in advance that certain phonemes

567 (e.g., non-sibilant fricatives like /f/ and / $/$ /) would be harder than others (stops) and our goal was

3 This is functionally equivalent to a logistic mixed effects model. 


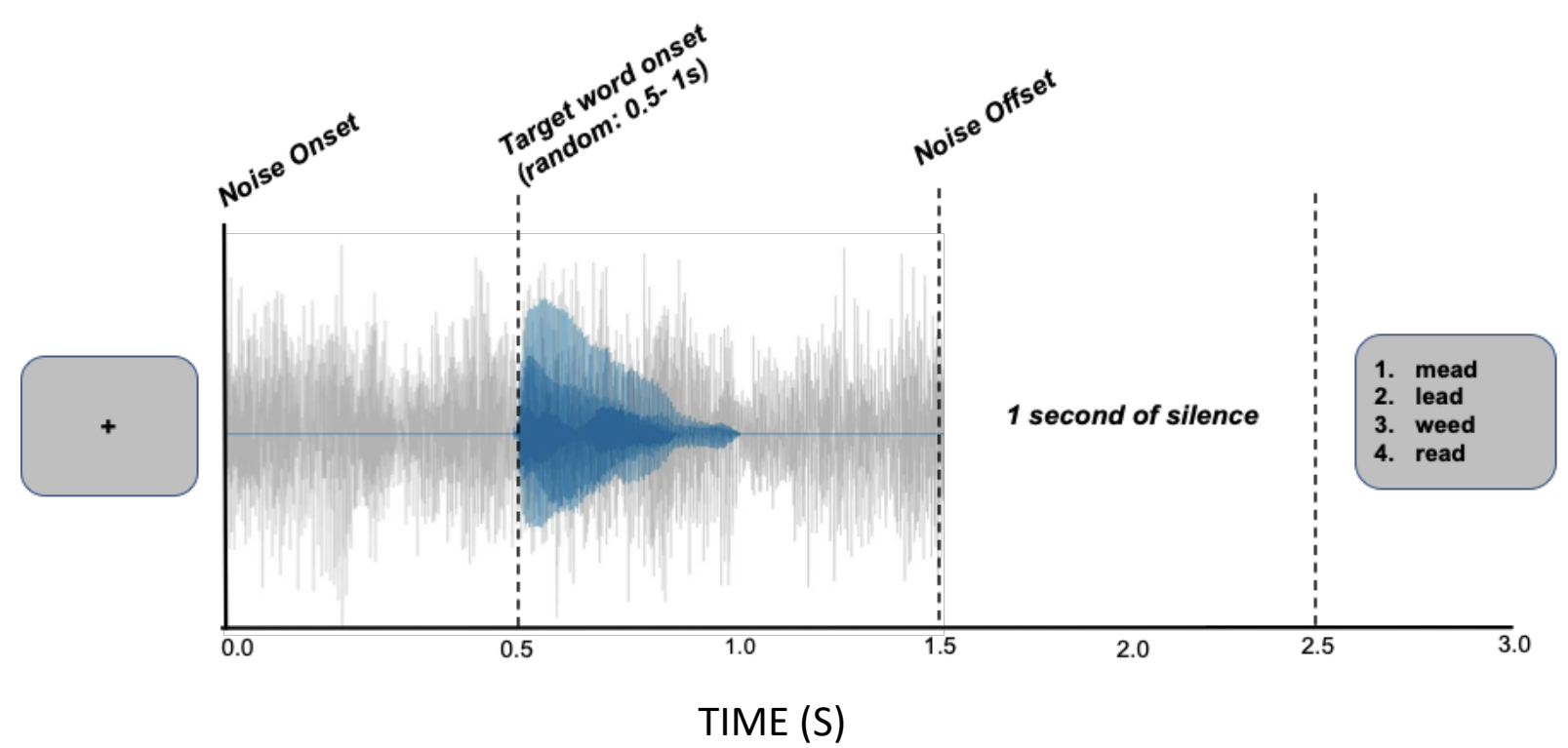

Figure 5. (Color online). Trial structure of the ITCP.

568 to ensure a uniform distribution of phonemes in the assessment (e.g., we did not want to exclude

569 all the non-sibilants, just those that were harder than non-sibilants should be in general).

570 We then used these item difficulty estimates from this model to eliminate sets that

571 contained outliers (items with extreme positive or negative difficulty estimates greater than +2 or

572 less than -2). Note that the fixed effects of this model did not include any information about the

573 difficulty expressed by the contrasts of the foils. Consequently, the easiness estimates likely

574 reflect in part variation due to the foils (e.g., a word with all multi-feature foils would appear as

575 easier than a word with single-feature foils). Thus, trimming out the extreme values helped

576 constrain the overall easiness (driven in part by the foils) to be similar across items.

577 The IRT analysis produced a set of 120 words (split into 30 sets) which simultaneously

578 balanced several factors: 1) roughly equal number of words for each consonant; 2) roughly equal

579 distribution of vowel contexts, by manner and place of articulation; 3) no item could appear in

580 more than one set; 4) sets maximized single-feature contrasts and were of roughly equal 
581 difficulty (e.g., the number of one-or-two feature contrasts) across different phonemes. Given

582 English language constraints, there is no perfect set that maximizes all of these constraints;

583 however, our goal was to come close. If a researcher seeks to use a subset of items or to

584 recombine them, we strongly urge them to respect these factors to ensure a uniform test.

\section{Final ITCP}

The final version of ITCP consisted of $120^{4} \mathrm{CVC}$ words, each produced by two male and two female speakers of midland American English. The words spanned 19 onset consonants (i.e.,

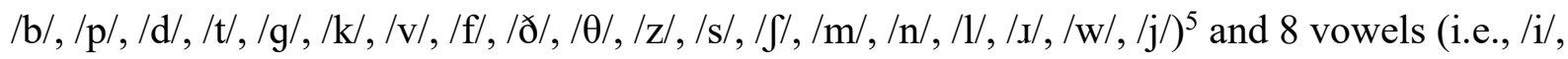

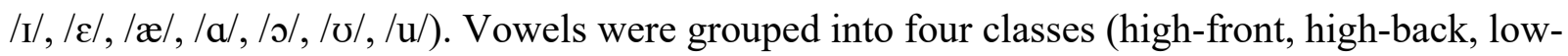

590 front, low-back), where class contains one or more nearby vowels (e.g., /æ/ and /ع/ were both

591 low-front). Within each phoneme $\times$ vowel combination, there is an average of 2 words, and most

592 phonemes appear with vowels in each of the four quadrants (Figure 4). The ITCP includes four

593 talkers, though each participant here was tested on only two; in practice, the ITCP could be

594 expanded by using three or even all four talkers or reduced by using a single talker ${ }^{6}$.

595 Consequently, the ITCP can range from 120 - 480 trials. Details about the number of trials

4 In the validation latch was used as a foil in lieu of thatch for the targets batch and match).

5 Four consonants were left out (e.g., /3/, /h/, /t $\mathrm{J} /, / \mathrm{d} z /$ ). $/ 3 /$ was excluded due to the small number of items available that contrast this phoneme word initially. $/ h /$ was left out because the initial development of the ITCP was for use in ERP experiments and it is too low amplitude and too variable to elicit reliable ERPs (though it should have been added back in when our goals were broadened). Finally, the two affricates $\left(/ t f /, / d_{3} /\right)$ were left out because many phonological analyses treat these as two phonemes - not one-and our long-term goal was to be able to measure performance on isolated dimensions.

6 Though we note that a single talker may create "process of elimination" task demands. We discuss these (and how to potentially get around them) in the "design principles" document on the OSF site. 
tapping specific phonological dimensions (e.g., voicing) are shown in Figure 2; and the number of trials for each vowel context are shown in Figure 4. The distribution of possible target-foil pairs is included in Appendix A, along with a confusion matrix (Appendix B) highlighting the probability of an error given the target phoneme across all participants in Session 1.

\section{Pilot Experiment 2}

602

To ensure performance was not due to differences in stimulus audibility, we ran a second

603 pilot study $(N=45)$ assessing performance on the final items in quiet. This was run online using 604 the Gorilla platform (gorilla.sc; Anwyl-Irvine et al., 2019) and the Prolific (prolific.co)

605 recruitment platform. Methods were identical to those used in the validation study below (except 606 for the absence of noise). Participants were reimbursed for their time.

607 The mean level of performance in silence (proportion correct) across all speakers and 608 items was near ceiling $(M=.95, S D=.08$, range $=.49-1.00)$. The mean level of performance 609 (proportion correct) across all items was also high $(M=.95, S D=.05$, range $.73-1.00)$. The 610 mean performance between speakers was also high: F1 $(M=.94, S D=.23)$; F2 $(M=.96, S D=$ $611.19), \mathrm{M} 1(M=.96, S D=.18), \mathrm{M} 2(M=.94, S D=.24)$.

\section{III. Validation Study}

613 A. Overview

614 The empirical study reported here is a validation of the complete assessment. We tested 615 normal-hearing listeners to establish the reliability and validity of the ITCP and gather estimates 616 of item-level easiness. We assessed convergent validity by relating the ITCP to two other speech 617 measures (tested in noise): CNC (a single word open-set task) and AzBio (an open-set sentence618 based task; Spahr et al., 2012). Both the CNC and AzBio are widely used clinically with hearing- 
619 impaired populations (e.g., The Minimum Speech Test Battery for Adult Cochlear Implant Users 620 [MSTB], Luxford, 2011) as measures of speech perception ability. Further, the CNC and AzBio

621 tests are both used by the University of Iowa Cochlear Implant research team (with which the

622 ITCP will be used as part of a long-running research program). These validation measures allow 623 the ITCP to be mapped to current clinical measures and could help unify research and clinical 624 assessments.

All participants completed the ITCP, the CNC words (100 items), and the AzBio (1 list of

62620 sentences). To assess test-retest reliability, we examined ITCP performance in a smaller 627 group of listeners across two sessions spaced one week apart. Data collection took place online 628 using Gorilla (Anwyl-Irvine et al., 2019). While online testing offers some specific advantages 629 worth noting, the advantages and limitations will be discussed before turning to the methods.

\section{Online testing}

In recent years, many labs have begun to explore online platforms to build and run

632 experiments. In the lab, collecting data can be time-consuming and expensive and tedious.

633 However, online data collection poses several challenges for auditory work. Although web

634 technologies can effectively standardize the presentation of simple visual stimuli (such as words

635 or images), researchers have significantly less control over the quality and loudness of audio

636 stimuli and, historically, the precision of the onset time of the stimulus. In-lab research typically

637 entails a careful specification of the signal-to-noise ratio and the overall amplitude of stimuli.

638 Stimuli are typically presented to all participants via the same high-quality headphones or 639 speakers and in a sound-attenuating chamber with no visual distractions. For online studies, 640 audio hardware varies among users, and it is almost impossible to ensure that auditory stimuli are 
641 presented at a consistent volume across listeners ${ }^{7}$. Also, researchers have no control over the

642 acoustic environment where the testing takes place; therefore, most participants will be listening

643 in settings that are less neutral than a sound-attenuated booth.

644 Despite giving up experimental control in some areas, online auditory processing studies

645 show high fidelity to lab-based studies. Cooke et al. (2011), for instance, found that laboratory

646 and online participants were similarly affected by changes in signal-to-noise ratio and the type of

647 masker noise (i.e., multi-talker babble, speech-shaped noise, etc.). In addition, Slote and Strand

648 (2016) demonstrated a correlation between online and in-lab measures of both speech-in-noise

649 identification (Experiment 1) and response times in an auditory lexical decision task (Experiment

650 2). Obtaining this kind of reliability requires techniques to ensure 1) that subjects are wearing

651 headphones; 2) that the sound card is of sufficient quality; and 3) that the stimuli are audible.

652 With these solutions in hand, online testing may offer many advantages for a project like

653 this. First, online data collection allows for more diverse participants than university participant

654 pools, and these participants are willing to participate for less compensation as they can

655 participate at the time and place of their choosing (Paolacci et al., 2010). Online data collection

656 also allows researchers to recruit a large number of participants at a quicker rate than traditional

657 laboratory settings — even during periods when recruiting undergraduates is difficult, such as

658 between terms (Crump et al., 2013; Grootswager, 2020; Mason \& Suri, 2011). A larger and more

659 diverse group of participants can increase between-subject variance, which helps correlational

660 studies like this one and leads to a more generalizable sample.

661 Second, given that online users never interact with an experimenter and have no

\footnotetext{
${ }^{7}$ Although their ability to adjust the volume may make it such that online users are better matched at a subjective level of loudness (e.g., the level at which they feel like they can perform the task accurately) even if they are not matched objectively.
} 
662 preconceptions about the kinds of studies being done in a particular research lab, online data

663 collection may help avoid experimenter bias or effects of participant expectation.

664 Finally, and most importantly, online listeners' diverse listening situations may be a

665 strength for this study. The goal of this study is not to estimate a psychophysical threshold but to 666 ask how variation in performance in the ITCP relates to variation in other tests and to itself. In

667 this context, diverse listening situations may yield a better approximation of general

668 confusability and intelligibility than do data obtained from stimuli presented in a carefully

669 controlled setting. This is because the same issues driving diversity in listening conditions (e.g.,

670 sound quality, background environment) apply in the ITCP and the CNC/AzBio sessions. Thus,

671 these results may generalize to a variety of sound presentation formats, overall amplitudes, and

672 so forth, and as a result, the conclusions drawn from online experimentation may be more robust 673 and generalizable to natural settings than lab-collected findings.

\section{B. Methods}

\section{Participants}

Three hundred and eighteen participants signed up online using the Gorilla hosting and

677 experiment platform (gorilla.sc; Anwyl-Irvine et al., 2019). Participants were recruited via

678 Prolific (prolific.co) and reimbursed for their time. Using pre-screening questionnaires on

679 Prolific, we limited our sample to participants from the USA, aged between 18-47, and native

680 English speakers. Participants were also screened to ensure they wore headphones during the

681 task and had no history of self-reported neurological, visual, or auditory issues. After rejecting

682 participants that did not pass the headphones screener $(n=50)$ and eliminating from analysis any

683 individuals that did not complete all tests within a session $(n=69)$, the final sample size for

684 Session 1 was 199 participants. Ninety-eight of these participants successfully returned to 
685 complete Session 2 . A sensitivity analysis indicated that this sample size $(n=98)$ provided 686 adequate power $(0.80)$ to detect a small effect size $(r=0.28)$ or larger. The demographic

687 information for those that completed both sessions is shown in Table 1.

688 2. Materials

689 All tests were programmed using Gorilla and can be viewed at:

690 https://gorilla.sc/openmaterials/97811. Audio files were converted to MP3 at $320 \mathrm{~kb} / \mathrm{s}$. The 691 master copies (in WAV format) are available on the OSF site (https://osf.io/hycdu/).

692 a. Headphones screener. The headphone screener is a six-trial task taken from Woods et 693 al. (2017). On each trial, three tones of the same frequency and duration were

Table 1. Demographic Variables $(\mathrm{N}=97)$

\begin{tabular}{ll} 
Factor & M / N (range / \%) \\
\hline Age & 27 years $(23,35)$ \\
Gender & $48(49 \%)$ \\
Female & $48(49 \%)$ \\
Male & $1(1.0 \%)$ \\
Other & $9(22 \%)$ \\
Race & $11(27 \%)$ \\
Asian & \\
Black or African & $21(51 \%)$ \\
American & 56 \\
White & \\
Unknown &
\end{tabular}
presented sequentially. One tone had a lower amplitude than the other two tones. Tones were presented in stereo, but the tones in the left and right channels were 180 degrees out of phase across stereo channels - in free field, these sounds should cancel out or create distortion, whereas they will be perfectly clear over headphones. The listener picked which of the three tones was the quietest. Performance is generally at the ceiling when wearing headphones, but poor when listening in the free field (due to phase cancellation). 
b. ITCP. Each participant was randomized to one of four counterbalanced lists by the experimental control software. Each list contained 240 trials $^{8}$, with each word spoken by one male and one female talker, with each list containing a different combination of male and female talkers. For reliability testing, the second test for a given subject used a list that tested the two talkers not used in Session 1. We used the same procedure as in the pilot (Figure 5 for a schematic). A break was provided after each block of 50 trials. Before the experiment proper, participants were given eight practice trials, with feedback, to familiarize themselves with the task. was used as a proximal validity measure. It consists of 500 test words organized into 50-word lists (10 lists in total) with a frequency-weighted consonant distribution. For our validation study, we used Lists 1 and 4 for a total of 100 individual words.

Words were presented at -2.5 signal-to-noise ratio (dB SNR) in multi-talker babble. On each trial, individuals saw a fixation cross for $500 \mathrm{~ms}$ and then heard a word, spoken by a single male speaker in the same multi-speaker babble used in the ITCP task. At the offset of the audio, participants typed the word they heard before going on to the next screen. Breaks were provided every 50 trials. Words were presented in a random order. Written responses were scored using the $l r d$ package in $\mathrm{R}$ (Maxwell et al., 2020). This package automates scoring of single-word responses. Responses were correct if the typed response completely matched the target. d. AzBio sentence recognition. The AzBio sentences were used to assess validity with a

8 We also had 10 extra trials that served to eliminate process of elimination bias. Specifically, we repeated cell, chute, cook, gab, lock, lot, nab, rock, shore, and thong. 
more distal measure. It consists of 1000 sentences recorded from two male and two female talkers (Spahr et al., 2012). For our validation study, we presented AzBio List 1 (20 sentences, in random order) — at -2.0 dB SNR in multi-talker babble. On each trial, participants first saw a fixation cross for $500 \mathrm{~ms}$ and then heard a sentence, spoken by either a male or female talker in the same multi-speaker babble used in the ITCP and CNC tests. At the offset of the audio, participants typed the words in the sentence. Written responses were hand-scored by two independent reviewers. We employed a single-score, absolute agreement, two-way random effects model, and found high agreement among the two raters, $\operatorname{ICC}(\mathrm{A}, 1)=.989,95 \% \mathrm{CI}=[.988, .990]$. As a result, only the scores from the first reviewer were used. During scoring, a trial was considered correct if all words in the sentence were correct. Individual words were correct if they were the same word as in the target sentence with exceptions made for contractions (e.g., typed response of "it's" for actual words "it is") and for misspellings with the same meaning (e.g., typed "pak" for "pack"). English); gender; race; age; if they had ever been diagnosed with hearing loss, neurological disorder, or have vision problems; if they had trouble hearing a sample audio file (yes or no); and to report the brand of headphones used during the task.

\section{General procedure}

742 completed (in order) the headphones screener, ITCP, and CNC. In Session 2, they completed (in

743 order) the headphones screener, ITCP, AzBio, and the basic demographics questionnaire. 
information about the study to decide whether they wished to participate. At this point, participants provided informed consent. After consenting, participants were presented with noise and told to adjust the volume until it was at a comfortable level. Next, participants were given the headphones screening test. Participants had two attempts to pass the headphones screener. If they did not pass on the second attempt, they were excluded from the experiment. If the participant passed the headphones screening, they next completed the ITCP followed by the CNC.

b. Session 2. Seven days later, the Gorilla platform sent email reminders to participants for the second session. After logging in, participants completed the headphones Session 1. After completing the ITCP, participants completed the AzBio task. At the end of Session 2, participants took the demographics questionnaire.

\section{$758 \quad$ IV. Results}

Our analysis starts by reporting descriptive statistics for overall performance. Next, we

760 conducted an item analysis to obtain raw difficulty/easiness estimates for reporting with these

761 items. Following this, we conducted a similar analysis which accounted for known sources of

762 accuracy differences (e.g., differences among consonants, talkers, vowel contexts, and foil

763 composition). This was intended to both evaluate our materials by asking if accuracy was

764 systematically affected by the expected factors, and to identify the difficulty/easiness of each

765 item relative to these factors (for use in future iterations of the ITCP). Finally, we report validity

766 (correlations against the other speech-in-noise measures) and test/retest reliability.

\section{$767 \quad$ Overall ITCP performance}


The mean performance (proportion correct) across session 1 ranged from .32 to $.89(M=$

$769.73, S D=.08)$ with similar difficulty for session $2(M=.74, S D=.10$; Range: .43 to .90$)$.

\section{A. Individual item analysis}

The words in the ITCP were evaluated to assess the relative easiness/difficulty of the individual words contained in the test (see Appendix $\mathrm{C}$ for base easiness values). A multilevel 1PL Rasch model was constructed (model formula in glmer [R] notation: accuracy $(1 \mid$ Participant $)+(1 \mid$ item $)$. The code for these analyses is available on our OSF page (https://osf.io/yunhp/). The analyses used all available data from session $1(n=199)$. Each item and its easiness score can be found on our OSF page here (https://osf.io/n496k/). The items and their characteristics can be used for secondary analyses or for counterbalancing new ITCP lists. Note that these easiness estimates do not account for systematic differences among talkers, phonemes, or contexts; thus, they reflect the easiness of entire classes of sounds (e.g., nonsibilants are harder than other consonants).

\section{B. Participant and item level analysis}

Next, we constructed a more complex version of the prior model, which included fixed effects that capture several known sources of variance in trial-by-trial performance: phonetic factors, coarticulatory contexts, differences among talkers, and foil composition. This model included random item-level and participant-level effects. The easiness values conditionalized on these factors are presented in Appendix C.

With this more complex model, we had two goals. First, by estimating the item-level random effects, we estimate the relative easiness/difficulty of the individual words relative to the factors captured by the fixed effects. Here, the fixed effects code for the systematic differences among phonemes (etc.), and the random effects capture the relative easiness after accounting for 
791 this. Given this goal, the particular structure of the fixed effects matters less than whether or not 792 they capture differences among the phonemes, context, and foil composition. The resulting 793 easiness estimates assess the degree to which an item is as easy or difficult as expected for that

794 particular phoneme/talker/context. These are probably the most relevant easiness/difficulty 795 estimates to balance when using the ITCP items for other purposes.

796 Second, by evaluating the significance of the fixed effects, we asked if factors like target 797 voicing, vowel context, or foil composition affected performance. It is important to note that 798 with respect to this goal, these analyses are exploratory and do not represent all the factors that 799 could influence speech perception, nor do they capture a perfect phonological analysis of these

800 words. Rather in developing these models, our goal was to derive a reasonable model which 801 avoided collinearity, had a relatively small number of terms, and permitted some inferences 802 about systematic factors of interest.

803 We considered several possible coding schemes to capture between-phoneme differences.

804 Recall that our goal was to ask if a specific token was easier or harder than expected for that 805 phoneme class. That ruled out an acoustic scheme as these acoustic cues would need to be 806 measured from the specific tokens. There is a variety of phonological options, but many of these 807 are isomorphic to each other (meaning they would capture the same cross-phoneme variance), 808 thus we strove for simplicity. We considered a simplistic fixed effects scheme that simply 809 crosses factors like place of articulation and voicing. However, this is not statistically sound.

810 The phonology of English is not fully balanced in many ways -- there are no voiceless

811 approximant liquids, nasals, or glides; and the post-alveolar and interdental places of articulation 812 only appear in fricatives. Consequently, any set of fixed effects based on broad phonological 813 features will have a high degree of collinearity. A voicing contrast, for example would be highly 
814 correlated with a manner contrast (since there are no voiceless sonorants). Such collinearity is a

815 serious issue in fitting mixed-effects or regression models. Consequently, we designed a fixed-

816 effects coding scheme that captured orthogonal contrasts where possible (e.g., place and voicing

817 within stop consonants) while capturing other consonant differences in a way that would not be

818 confounded. For example, the voicing contrast was only active (-1 or 1$)$ for stops and fricatives

819 and was set to 0 for sonorants.

$820 \quad$ Fixed effects included the following contrast codes $(-1,0,1)$. Consonant voicing was

821 coded as +1 for voiced, -1 for voiceless, and 0 for sonorants (since there are no voiceless

822 phonemes at these manners). Manner of articulation was coded in two codes: stops (stop or not

$823+/-1$ ), fricatives (fricative or not, $+/-1$ ), with sonorants as a reference category. Place of

824 articulation for stop consonants was coded with two contrasts: bilabial or not $(+/-1)$, and velar or 825 not $(+/-1)$, with alveolar as a reference category, and set to 0 for fricatives and sonorants. Place

826 of articulation within fricatives was coded similarly with three contrasts (labio-dentals,

827 interdentals and alveolars) with post-alveolars as a reference category and set to 0 for all other

828 contrasts. The six sonorants were coded with five contrast codes. These were set to 0 for stops

829 and fricatives. They were +1 if the trial tested one specific sonorant (e.g., $/ \mathrm{m} /, / \mathrm{n} /, / 1 /, / \mathrm{d} /, / \mathrm{j} /$; with

$830 \mathrm{~K} / \mathrm{w} / \mathrm{held}$ out as the reference category) and -1 if it did not (e.g., the $/ \mathrm{m} /$ contrast was set to +1 if

831 the sound was an $/ \mathrm{m} /$, and -1 for $/ \mathrm{n}, 1, \mathrm{r}$, etc. and 0 otherwise). Note that the vectors of fixed

832 effects were orthogonal across phonemes; consequently, they capture similar variance to a set of

833 orthogonal dummy codes allowing us to focus on the relative easiness of each token.

834 In addition to coding these phonological factors, we included talker type (F1, F2, M1,

835 M2), vowel type (high front, high back, low front, and low back) as categorical variables. Lastly,

836 we controlled for the influence of foils on speech recognition by computing the number of foils 
837 that differed only by voicing, manner, or place in each set (see Figure 2). The model formula is 838 in (1), in notation of the lmer R package. When fitting the models, we used the Optimx

839 optimizer. Statistics for the full model are presented in Table 2.

840

841

842

843

844

845

846

847

848

$$
\begin{aligned}
\text { correct } \sim \sim & \text { isStop }+ \text { isFric }+ \text { Voicing }+ \\
& \text { labialstop }+ \text { velarstop }+ \\
& \text { labiodentalfric }+ \text { interdentalfric }+ \text { AlveolarFric }+ \\
& \text { son1 }+ \text { son2 }+ \text { son3 }+ \text { son } 4+\text { son } 5+ \\
& \text { talker }+ \text { vowel }+ \\
& \text { NumMannerFoils }+ \text { NumVoicingFoils }+ \text { NumPlaceFoils }
\end{aligned}
$$

Table 2. Model Summary for Full Model

\begin{tabular}{lccc}
\hline Variable & $\chi^{2}$ & DF & $\boldsymbol{p}$ \\
\hline IsStop & 0.186 & 1 & .666 \\
IsFric & 0.067 & 1 & .796 \\
Voicing & 0.610 & 1 & .435 \\
Labialstop & 10.235 & 1 & 0.001 \\
Velarstop & 2.712 & 1 & 0.100 \\
Labiodentalfric & 35.920 & 1 & $<.001$ \\
Interdentalfric & 41.29 & 1 & $<.001$ \\
AlveolarFric & 0.224 & 1 & 0.635 \\
Sonorant1 & 0.005 & 1 & 0.946 \\
Sonorant2 & 4.469 & 1 & 0.035 \\
Sonorant3 & 0.228 & 1 & 0.635 \\
Sonorant4 & 0.010 & 1 & 0.917 \\
Sonorant5 & 1.69 & 1 & 0.193 \\
Talker & 106.73 & 3 & $<.001$ \\
Vowel & 33.78 & 3 & $<.001$ \\
NumVoicingFoils & 0.103 & 1 & 0.749 \\
NumPlaceFoils & 2.60 & 1 & 0.107 \\
NumMannerFoils & 1.06 & 1 & 0.303 \\
\hline
\end{tabular}

849

850 The primary goal of this model was to assess item easiness (the random intercept of each item)

851 relative to these fixed effects. However, as an exploratory goal, we evaluated the significance of

852 the fixed effects to validate that the expected patterns of difficulty would be observed.

853 Significance was assessed via likelihood ratio tests wherein the full model was compared to a 
A

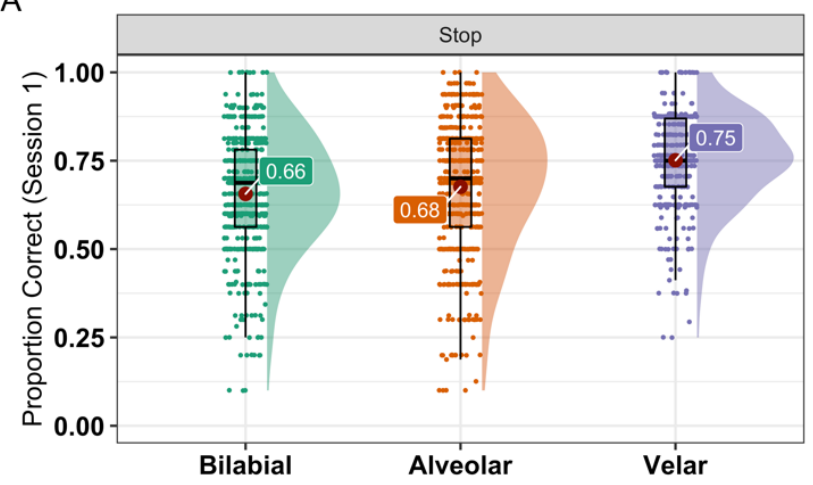

B

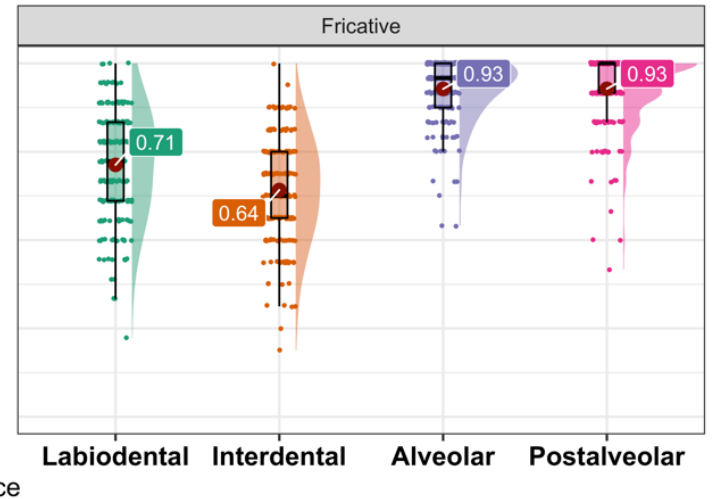

C

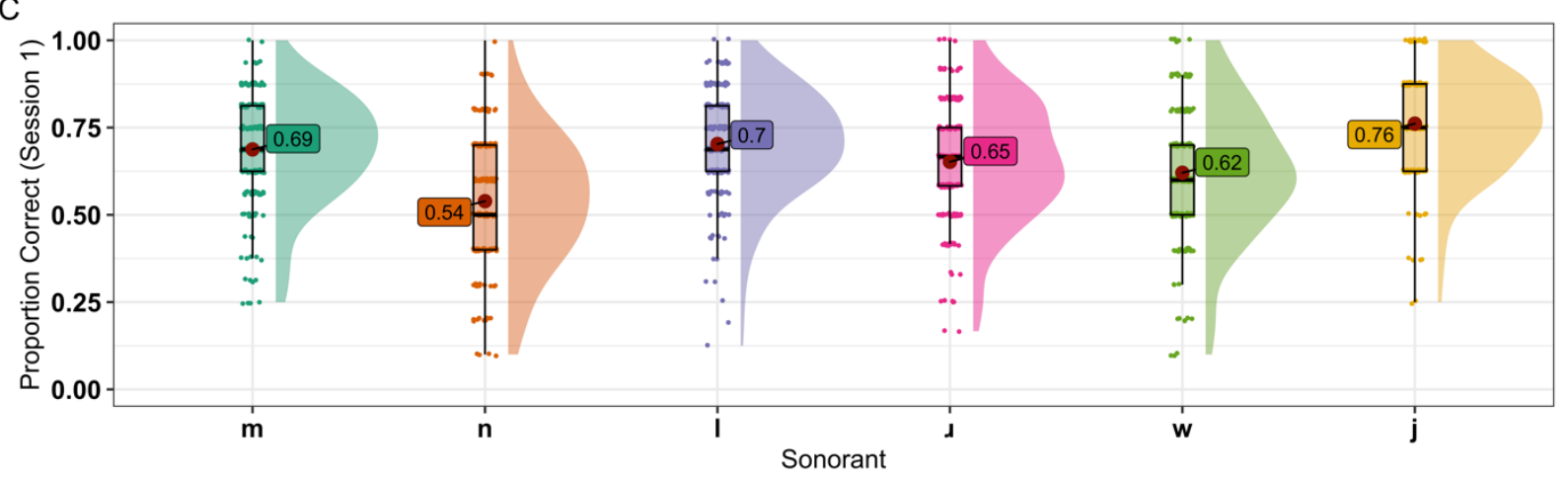

Figure 6. Raincloud plots (Allen et al., 2019) depicting results for Session 1. A. Raincloud plots for stops. B. Raincloud plots for fricatives. C. Raincloud plots for sonorants. Shown are by-participant accuracies (dots), medians and quartiles (box plots), grand means (red dots), and distributions of accuracy scores (half violin kernel density plots).

857 reduced model (with the effect of interest removed). This is similar to how a main effect in a traditional analysis of variance (ANOVA) would be interpreted. There is some debate about appropriate random effects structures for fixed effects (Barr et al., 2014; Seedorff et al., 2020).

860 However, the complexity of the model prevented us from fitting random slopes of participants, so fixed effect evaluation should be treated as exploratory and anti-conservative.

We start by considering the item-level (phonetic features) of the words themselves. We

863 found no overall effect of manner of articulation $\left(\chi^{2}(2)=0.238, p=.888\right)$; that is, there was no

864 evidence stops, fricatives, and sonorants differed as a whole. There was also no reliable effect of 865 voicing $\left(\chi^{2}(1)=0.61, p=.434\right)$ within the fricatives and stops. 
867 effect $\left(\chi^{2}(2)=9.84, p=.007\right.$; Figure 6). This was because bilabials $\left(\chi^{2}(1)=10.24, p<.001\right)$ were

868 harder to recognize on average. Similarly, there was also an overall effect of place within

869 fricatives $\left(\chi^{2}(3)=57.36, p<.001\right)$. This was because labiodentals $\left(\chi^{2}(1)=35.92, p<.001\right)$, and

870 interdentals $\left(\chi^{2}(1)=41.29, p<.001\right)$ were harder to recognize on average - this was quite

871 expected given prior work (McMurray and Jongman, 2011). There was a significant effect of

872 consonant type within the sonorants $\left(\chi^{2}(5)=12.47, p=.029\right)$. This was driven by $/ \mathrm{n} / \mathrm{relative}$ to

873 other sonorants, $\chi^{2}(1)=4.47, p=.035$. The finding that $/ \mathrm{n} / \mathrm{sounds}$ are more difficult maps onto

874 some prior work on nasality and speech perception (e.g., Beddor \& Krakow, 1999).

875 Next, we turn to the two context factors. The effect of Talker was significant (Figure 7;

$\left.876 \chi^{2}(3)=106.19, \mathrm{p}<.001\right)$. Post-hoc comparisons (Tukey-adjusted) using emmeans (Version

877 1.5.0; Lenth, 2020) showed that target words spoken by F1 were less likely to be perceived

878 correctly than target words produced by $\mathrm{M} 1(O R=0.74, S E=0.05, \mathrm{z}=-7.89, \mathrm{p}<.001), \mathrm{M} 2(O R$

$879=0.72, S E=0.03, \mathrm{z}=-8.73, \mathrm{p}<.001)$, and F2 $(O R=0.71, S E=0.03, \mathrm{z}=-8.04, \mathrm{p}<.001)$. All

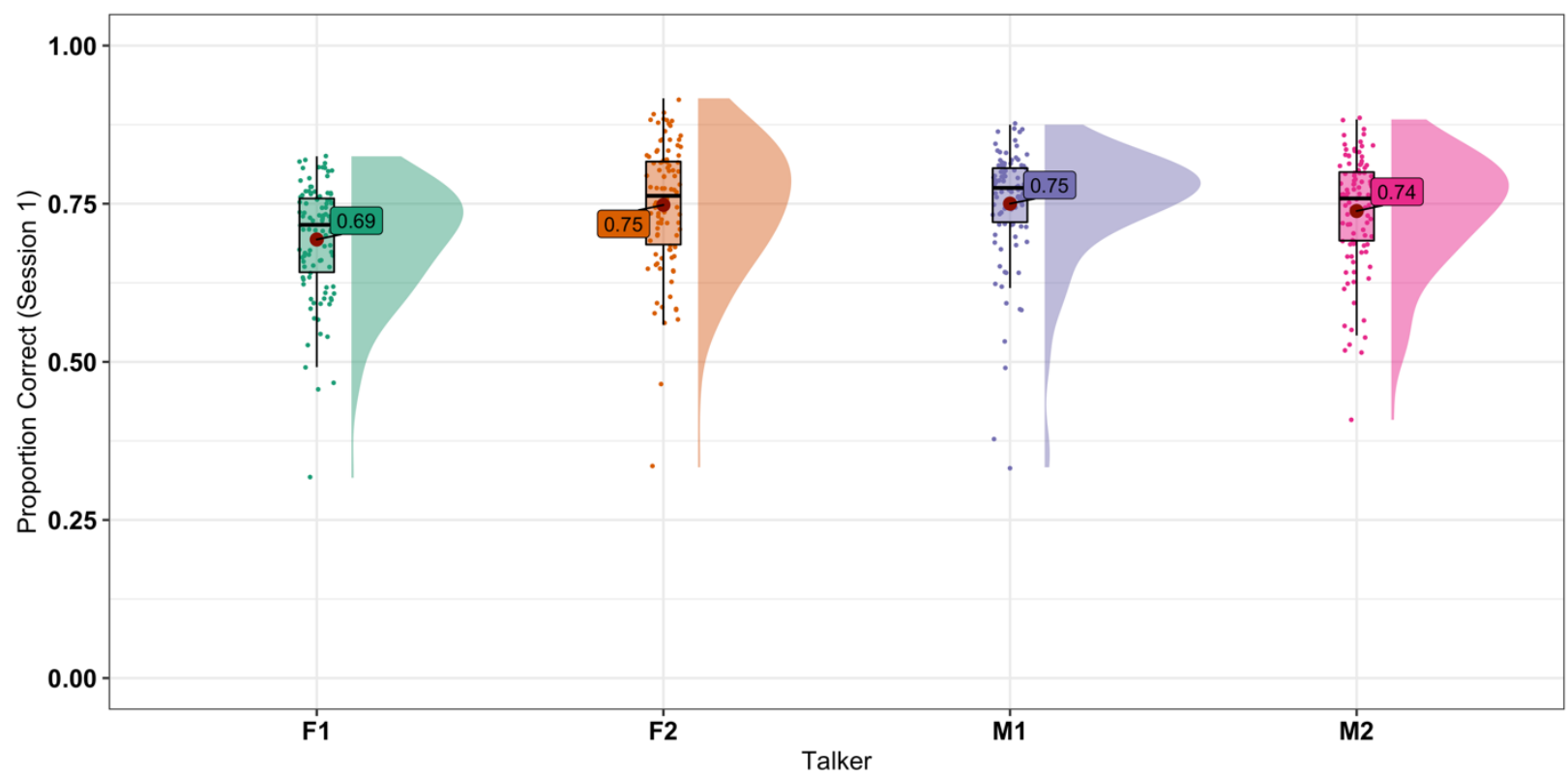

Figure 7. (Color online). Raincloud plots (Allen et al., 2019) depicting proportion correct during Session 1 as a function of Talker. Shown are by-participant accuracies (dots), medians and quartiles (box plots), grand means (red dots), and distributions of accuracy scores (half violin kernel density plots). 
other talker contrasts were not significant.

Vowel type (Figure 8 ) was also significant $\left(\chi^{2}(3)=29.90, p<.001\right)$. Post-hoc tests

882

883

884

885

886

887

888

889

890

891

892

revealed consonants before low back vowels were harder to recognize than consonants before high back $(O R=0.42, S E=0.46, z=-4.88, p<.001)$, high front $(O R=0.41, S E=0.43, z=-$

$5.16, p<.001))$, and low front vowels $(O R=0.64, S E=0.12, \mathrm{z}=-2.45, p=.027)$.

Lastly, the three foil contrasts did not affect perception of the target, $p s>.11$.

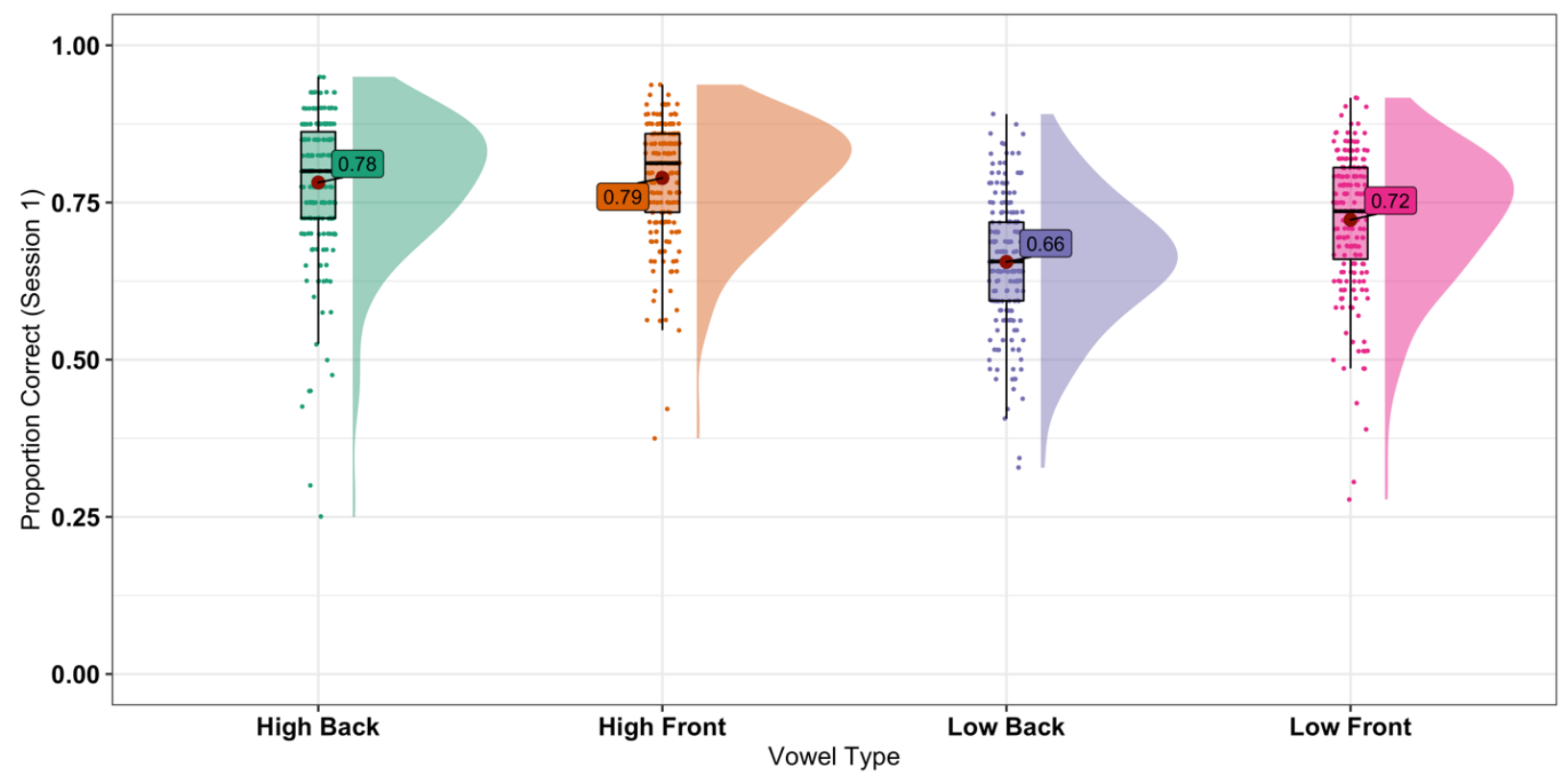

Figure 8. (Color online). Raincloud plots depicting proportion correct during Session 1 as a function of vowel quadrant. Shown are by-participant accuracies (dots), medians and quartiles (box plots), grand means (red dots), and distributions of accuracy scores (half violin kernel density plots).

\section{Test-retest reliability}

Finally, we turn to the most important goal of this project: establishing the reliability and validity of the ITCP. There was no overall accuracy difference between Sessions 1 and $2, \mathrm{M}_{\text {diff }}=$ $-.002, t(97)=-0.319, S E=0.008, p=.750$. This validates the efficacy of the design to prevent learning effects (the use of all four items in a set, the lack of repetition of individual exemplars, use of multiple repetitions to avoid process of elimination strategies). 
A $\quad t_{\text {Student }}(96)=8.82, p=5.07 \mathrm{e}-14, \hat{\rho}_{p b}=0.67, \mathrm{Cl}_{95 \%}[0.54,0.77], n_{\text {pairs }}=98$

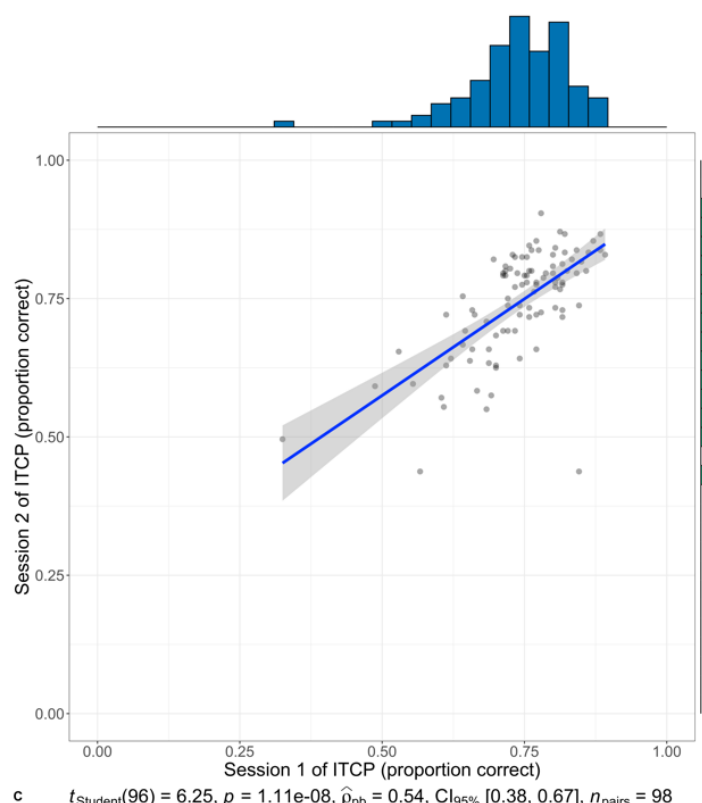

c $\quad t_{\text {Student }}(96)=6.25, p=1.11 \mathrm{e}-08, \hat{\rho}_{p b}=0.54, \mathrm{Cl}_{95 \%}[0.38,0.67], n_{\text {pairs }}=98$
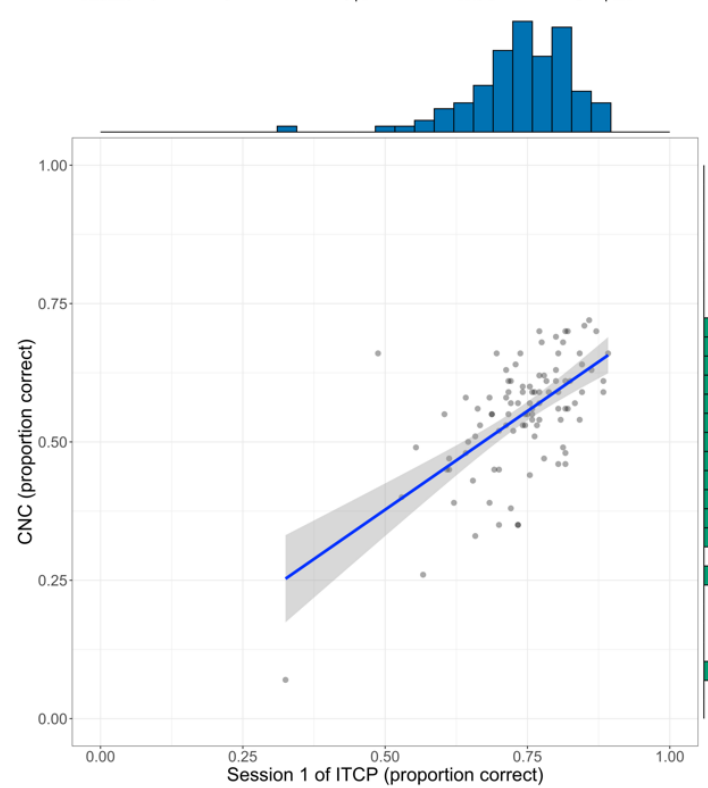

B $\quad t_{\text {Student }}(96)=7.10, p=2.12 \mathrm{e}-10, \hat{\rho}_{\mathrm{pb}}=0.59, \mathrm{Cl}_{95 \%}[0.44,0.70], n_{\text {pairs }}=98$
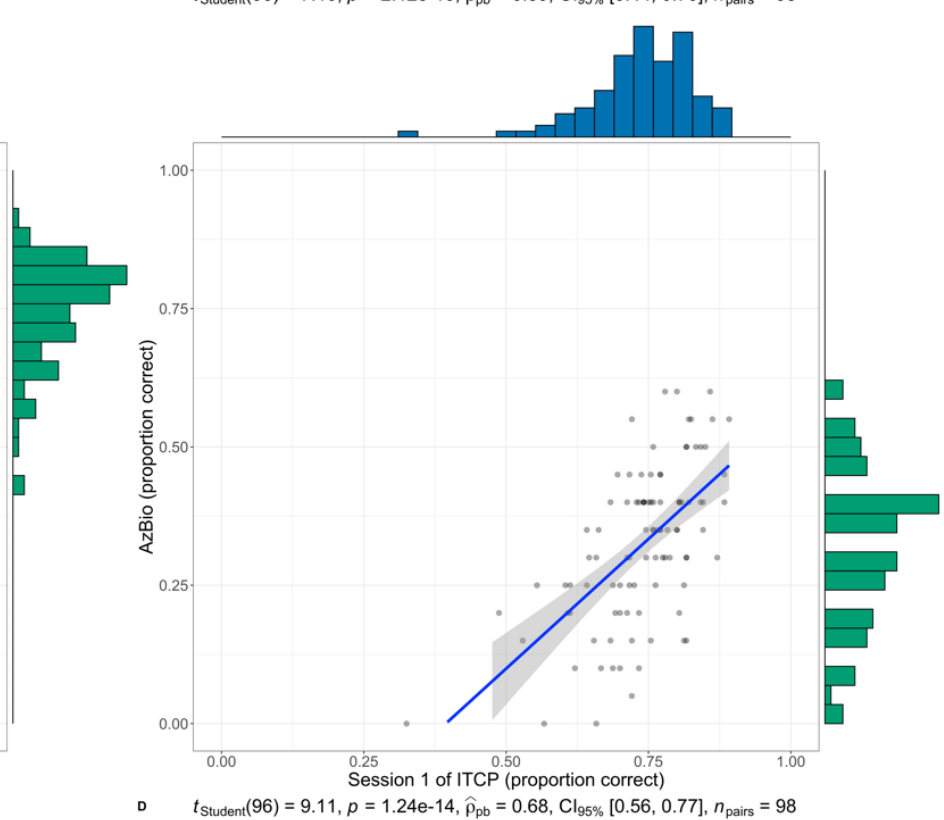

D $\quad t_{\text {Student }}(96)=9.11, p=1.24 \mathrm{e}-14, \widehat{\rho}_{\mathrm{pb}}=0.68, \mathrm{Cl}_{95 \%}[0.56,0.77], n_{\text {pairs }}=98$

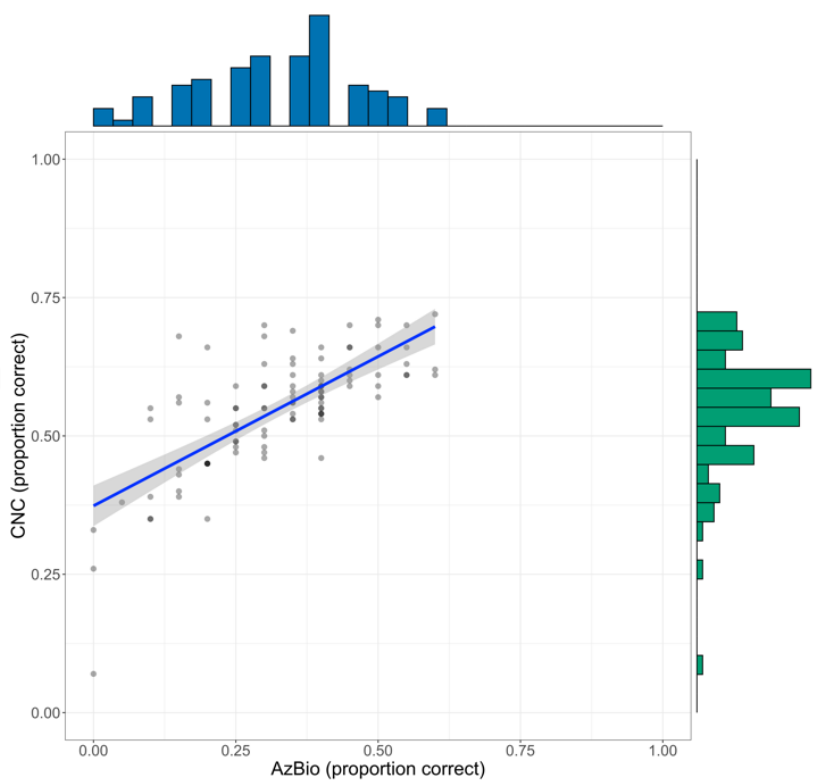

Figure 9. (Color online). A) Scatter plot of proportion correct between Session 1 and Session 2 of the ITCP, along with distribution of the data (histograms). B) Scatter plot of proportion correct between Session 1 of the ITCP and AzBio, along with distribution of the data (histograms). C) Scatter plot of proportion correct between Session 1 of the ITCP and CNC, along with distribution of the data (histograms). D) Scatter plot of proportion correct between AzBio and CNC, along with distribution of the data (histograms). Plots were created with ggstatsplot (Patil, 2021).

We next considered test-retest reliability of the ITCP. A scatterplot of Session 1 scores

895 and Session 2 scores can be seen in Figure 9a which shows a close relationship between

896 performance on the two sessions. We computed a numerical estimate of reliability using the 
897 intraclass correlation coefficient (ICC; Koo \& Li, 2016), estimated using the irr R package (vers.

898 0.84.1; Gamer et al., 2019). ICC is widely used within psychometrics (see Matheson, 2018)

899 which assesses absolute agreement between repeated measures (Session 1 and 2). It contrasts

900 with the standard correlation which reflects only predictability. Ninety-eight participants

901 completed the ITCP twice. We used an average-score, absolute agreement, two-way random

902 effects model, and we found good agreement among average percent correct across the two

903 sessions, $\operatorname{ICC}(\mathrm{A}, 2)=.80, F(97,97.2)=4.89, \mathrm{p}<.001,95 \% \mathrm{CI}=[.70, .86]$. See Figure $9 \mathrm{a}$ for

904 the correlation between Sessions 1 and 2.

905 D. Convergent validity

906

Finally, we examined the relationship between performance on Session 1 of the ITCP and

907 the AzBio (Figure 9B) and CNC (Figure 9C). Only listeners who completed all three measures

908 were included in the analysis $(n=98)$. We used a robust correlation method, the percentage-bend

909 correlation (Wilcox, 1994), which keeps outliers in the analysis but down weights them to

910 provide a better balance between Type 1 error and power. The use of the percentage-bend

911 correlation avoids researcher degrees of freedom in choosing a threshold for rejecting outliers.

912 We found a positive correlation between AzBio and ITCP, $r_{\mathrm{pb}}=.59,95 \%$ CI $[.44, .70], t(98)=$

$9137.10, p<.001$, see Figure $9 \mathrm{~B}^{9}$. We also found a positive correlation between CNC and ITCP, $r_{\mathrm{pb}}$

$914=.54,95 \% \mathrm{CI}[.38, .67], t(98)=6.56, p<.001$, see Figure $9 \mathrm{C}$.

\section{$915 \quad$ V. Discussion}

916 The goals of this project were twofold. First, we sought to create a single word, closed-set

917 task that could be used experimentally (with potential clinical utility down the road). Critically,

918 this task should 1) use real English words; 2) uniformly sample most consonants of English; 3)

\footnotetext{
${ }^{9}$ At the request of a reviewer, we also computed this correlation after scoring AzBio in terms of the average proportion of correct words. The correlation $\left(\mathrm{r}_{\mathrm{pb}}=.63\right)$ was very similar to the sentence-based result.
} 
919 control foils to ensure they played a minimal role in item-level performance and create

920 opportunities for assessing performance along specific phonetic dimensions down the road; and

9214 4) use words from a variety of talker and vowel/coarticulatory contexts to ensure robust

922 generalizability. Second, we sought to validate the task to ensure it possessed good psychometric

923 properties and showed the expected effects of phonetic and contextual factors.

924 A. Design of the ITCP

925 Our results support the first goal. The iterative development and final design of the ITCP

926 achieved its goals of uniformity and sampling (Figures 1-4; Appendix A), and the item-level

927 difficulty (both in absolute and relative terms) is available for finer-grained analysis.

928 Moreover, while the analysis of the experimentally manipulated factors showed few large

929 differences among consonants, there were robust effects of both talker and vowel context. The

930 effects of talker and vowel context supports the need for assessments that systematically sample

931 consonants from different talkers and from different coarticulatory contexts, as intelligibility can

932 vary markedly. For example, the difference between the highest $(M=.75)$ and lowest $(M=.69)$

933 performing talkers was approximately 6\%; see Figure 7) and the difference between the highest

$934(M=.79)$ and lowest $(M=.66)$ performing vowel was approximately $13 \%$ (see Figure 8 ) -

935 absolute differences whose sizes are quite meaningful in a closed set task.

936 While the effect of a talker is not unexpected due to factors like dialect, rate, or

937 articulation quality (though we attempted to select uniformly intelligible talkers with a standard

938 accent), the effect of vowel quadrant across context is less expected. It is well known that

939 coarticulatory context can shift consonant boundaries (e.g., Mann \& Repp, 1980). However, an

940 overall effect on intelligibility across most (or all) consonants has been reported previously (e.g.,

941 Donaldson, \& Kreft, 2006; McMurray \& Jongman, 2011; Singh \& Black, 1966; Wang \& Bilger, 
942 1973). We found that low vowels — particularly low back vowels (/a/ and /o/) — generally led to

943 consonants with lower intelligibility than consonants before high vowels. This may have been

944 because low vowels generally have higher first formants (closer to F2); this may have made it

945 more difficult to separate the formants to use them as independent sources of information (e.g.,

946 for place of articulation), particularly when F2 was low (as in back vowels). Indeed, we observed

947 consonants preceding low-back vowels (which have the highest F1 and the lowest F2) were

948 harder than consonants preceding low-front vowels (with a high F1, but also a higher F2).

949 However, future phonetic work should examine this more systematically to understand the

950 source of these intelligibility differences.

951 As a whole, however, these intelligibility differences support the need for assessments of

952 speech perception to test phonemes across multiple contexts (talker and coarticulatory) to ensure

953 that they robustly generalize to the variety of contexts listeners will encounter in the real world.

954 Finally, we note that a critical concern in the development of the ITCP was balancing the

955 difficulty of the foils across consonant classes - a critical concern about closed-set tests. Our

956 analyses with the composition of the foils relative to the target did not have significant effects.

957 This may be because variation in difficulty related to foil selection was minimized in two ways.

958 First, our automated procedures strove to balance this by selecting a set of items whose foils

959 were uniformly close. Second, after the first round of testing we used an IRT model to eliminate

960 sets that showed unexpectedly high or low performance (which was likely to be impacted by the

961 foils). This finding is encouraging as it suggests variation in performance across sounds in the

962 ITCP is likely due to the target sound, not, its foils.

963 B. Validity

964 Moving on to our second goal, our validation results showed that the ITCP is a valid and 
965 accurate task. First, the performance across phonemes were generally in accord with prior

966 estimates from laboratory experiments. For example, in stop consonants bilabials showed lower

967 intelligibility than others (e.g., Miller \& Nicely, 1955). Within fricatives, we observed lower

968 performance for non-sibilants than sibilants (e.g., McMurray \& Jongman, 2011). Overall, the

969 ITCP task appears to capture the kinds of patterns one would expect from lab-based studies.

970 We also obtained high test-retest reliability of the ITCP, suggesting that the task can be

971 re-administered after a week and possibly longer. Critically, there was no difference in mean

972 accuracy between the two sessions indicating that we were able to successfully prevent learning

973 by 1) using all four items within a set; 2) not repeating individual acoustic tokens; and 3)

974 repeating sets more than once (to avoid a process of elimination strategy). Following this, the

975 ITCP can be used for longitudinal testing in the same subjects. In this case, though, it may be

976 advisable to switch talkers as listeners can store and retain exemplars of words spoken in

977 particular voices over several days (c.f., Nygaard et al., 1995; see also Goldinger, 1996).

978 Finally, the ITCP showed strong positive correlations with other speech tests presented in

979 noise (i.e., CNC and AzBio). These tests were correlated with the ITCP at $r=.56(\mathrm{CNC})$ and $r=$

980.59 (AzBio). While not psychometrically adequate, these SiN tests should be contextualized in

981 light of the ITCP's test/retest reliability estimates of $r=.80$. As test/retest reliability is the

982 absolute highest correlation one could expect (a test should not be correlated with a completely

983 different measure more than itself), these validity estimates are fairly impressive (particularly if

984 the unknown reliability of the $\mathrm{CNC}$ and $\mathrm{AzBio}$ are taken into account). It is important to note

985 that these correlations were high even though the CNC and AzBio are open-set, suggesting that

986 the closed-set format did not suffer from ceiling effects (a concern with closed-set testing).

987 Moreover, it was particularly impressive that the correlation was as large as it was $(r=.59)$ with 
988 the sentence level test (AzBio), which was intended as a more distal measure. This suggests that 989 when properly designed, a closed-set word recognition test can give similar performance to the

990 currently more in-vogue open-set sentence tests. Further validation is, however, needed.

991 Collectively, the findings provide positive evidence for the basic application of the ITCP in 992 evaluating consonant perception in background noise.

\section{Availability}

We have made the materials for the ITCP freely available online, and we hope that other

995 labs can use it as a standardized set of speech perception materials for a variety of research

996 purposes. These materials can be recombined into smaller and larger tests. Still, we highly

997 caution researchers who wish to do this to pay attention to several design principles which are

998 essential for validity. This includes a uniform sampling of the space (e.g., roughly equal numbers

999 of trials for each consonant $\times$ vowel context $\times$ talker), use of all items in each set of four (so that

1000 subjects assume that all four items are equally likely), and the use of at least two repetitions of

1001 each item (so that subjects do not adopt a process of elimination strategy). To create your own

1002 version of the ITCP, we created a design principles document posted with the materials

1003 (https://osf.io/cyfpe/). The easiest approach, however, is to use all the items with two or more

1004 talkers, which gives 240 trials (2 talkers), 360 trials (3 talkers), or 480 trials (4 talkers). Our team

1005 is happy to consult with others on the appropriate way to do this.

\section{Limitations}

1007 The first limitation of this validation study is that the data were collected online. With the 1008 advancement of online behavioral research methods, it has become possible to test large samples 1009 of participants outside the laboratory with favorable results (e.g., Crump et al., 2013). However, 1010 for auditory testing, it becomes notoriously difficult to control important auditory factors (e.g., 
1011 sound quality) due to testing without an experimenter present in a contained environment with

1012 the proper audio equipment. The lack of control might call into question the validity of the 1013 current results.

1014 To partially mitigate some of these concerns, we took special precautions by including a

1015 headphones screener (Woods et al., 2017) and a simple hearing test at the beginning of the

1016 experiment to ensure participants could hear the stimuli and were wearing proper headphones. In

1017 addition, we asked participants to self-disclose their hearing status. These seemed to be adequate

1018 precautions. We observed high test-retest reliability and correlations with other well-established

1019 SiN measures. Moreover, online testing may be a strength for this study by generalizing over a

1020 wider range of possible listening conditions. Despite this, it is premature to argue that these data

1021 support the immediate use of ITCP. Future testing should be conducted to establish normative

1022 data and compare our online validation study results to the gold standard of laboratory testing.

1023 Initial evidence from an in-lab study pilot that showed similar results to those observed here.

1024 A second limitation of this validation study is its reliance entirely on normal hearing

1025 participants. We will eventually test a more heterogeneous population such as younger listeners,

1026 hearing impaired listeners, and hearing aid users with the ITCP. We will do so for two reasons.

1027 First, it is essential to show that these materials are equally valid and reliable for these users.

1028 Validity is perhaps the biggest concern as cochlear implants, hearing aids, and other devices can

1029 alter the spectral composition and fidelity of the input. Listeners who use these devices hear in a

1030 fundamentally different way than normal-hearing listeners, and it is essential to document that

1031 this validity and reliability extends to these conditions. Second, the pattern of easiness/difficulty

1032 across items may change in such listeners due to the same altered input. Thus, performance

1033 patterns across phonemes may not hold for such listeners. Third, it is crucial to collect normative 
1034 data with such populations to determine "how good is good"; at what level of ITCP performance

1035 should one be concerned that a given hearing aid user or CI user is likely to encounter problems.

1036 These normative data could inform clinical decision-making, although we note that most speech-

1037 in-noise tests currently in use in the clinic do not have such norms available. Thus, further

1038 research will be necessary to assess the validity and reliability of these materials for these

1039 specific applications and populations.

1040 As we described in the introduction, the ITCP was built with the goals of uniformly

1041 sampling the phonetic space and orthogonally varying phonetic properties along with contextual

1042 factors like articulatory context and vowels. At least from first principles, these goals suggest

1043 the ITCP should have been best suited for diagnosing and understanding specific areas of

1044 difficulty (or success) rather than predicting real-world outcomes. This may differ from

1045 assessments like the $\mathrm{CNC}$, which for example, attempts to match the frequency of testing a

1046 phoneme to its frequency of use (though see Martin, Champlin et al., 2000 for discussion of the

1047 difficulty of this). Nonetheless, the fact that we found strong correlations with CNC (and AzBio)

1048 suggests these fundamental differences in design principles may not result in huge differences.

1049 In light of these design goals, an important area for future work will be developing and

1050 validating sub-score measures for particular subclasses of sounds (e.g., fricatives) or particular

1051 contrasts (voicings). It may also be useful to incorporate some form of frequency weighting

1052 scheme into the scoring structure for greater predictive validity based on an individual's

1053 circumstances. Given the distribution of items and foils, this should be feasible with the present 1054 materials.

1055 The last limitation of the ITCP is that it is not a perfect assessment. That is, we tried our 1056 best to create a representative assessment that emulated real world situations by using multiple 
1057 speakers, uniformly sampling phonemic features, and balancing difficulty. In some instances, we

1058 were not successful due to the messy characteristics of the English language. Quite simply, all

1059 initial word consonants cannot exist in every category when dealing with real words (see

1060 Footnote 3 ). In other instances, we were clearly to blame (e.g., the lack of $/ \mathrm{h} /$ ). One concern is

1061 that the ITCP is not completely uniform. One solution to this problem might be to remove

1062 individual trials (e.g., an extra /b/ or /p/ trial) to achieve uniformity. We also note that the use of

1063 random effects models for assessment (De Boeck, 2008) could help. These models are

1064 structured similarly to our analysis (Equation 1). However, the goal is to estimate the random

1065 intercept of participants, which estimates each listeners latent ability, accounting for the fixed

1066 effects (e.g., talker, phoneme and item-level variance). These models are good at dealing with

1067 unbalanced designs and this helps them estimate individual participants' own ability level after

1068 accounting for an imbalance in the distribution of trials. Critically, this approach can also

1069 estimate participant ability relative to fixed effects such as consonant or talker - much like we

1070 used for the item analyses, potentially providing a robust estimate of subject ability.

1071 While the ITCP is not perfect, it accomplishes several goals not addressed by other

1072 measures. First, it improves upon some currently available closed-set tests by attempting to

1073 uniformly sample the phonemic space and balance difficulty of the foils. Second, it is

1074 completely open - all of the materials and data are available for public use. This open science

1075 approach is not widely adopted in the hearing sciences, but we hope it will enable other

1076 researchers to use this assessment and improve upon it.

1077 E. Conclusions

1078 A plethora of standardized measures exists to study speech perception. While the field

1079 has gradually emphasized sentence over word measures and open-set of over closed-set tests, 
1080 there is a need for closed-set single word tests. As we have described, these can isolate word

1081 recognition from more general sentence processing and memory processes; they can more

1082 uniformly sample the phonetic space and the range of phonetic and other contexts; they offer

1083 more precise timing for time-locking to cognitive neuroscience, pupillometry and eye-tracking

1084 measures; and a closed-set task can eliminate challenges involving speech production and is

1085 easier to code. As we've described, existing measures often fail to uniformly capture the space;

1086 they do not fully sample coarticulatory contexts and talkers, and they do not systematically

1087 control the nature of the foils to minimize the role of the response options.

1088 The ITCP is a single-word, closed-set task that uniformly samples the most frequently

1089 used isolated consonants and which uses response choices with equal proportions of phonetic

1090 features. This balance means that intelligibility estimates of the ITCP truly reflect virtually the

1091 entire set of word-initial isolated consonants in English, potentially enabling more diagnostic

1092 assessments of specific areas of difficulty. Our data show clear evidence of both validity and

1093 reliability. To facilitate the use of the ITCP (as well as conform to current open science

1094 practices), we have made all materials (audio files and scripts) along with important item

1095 characteristics freely and publicly available at our OSF page (https://osf.io/hycdu/). It is our hope

1096 that this will allow researchers to adopt and adapt the ITCP to fit their research needs. 


\section{Acknowledgments}

1111 The authors would like to thank Kristin Rooff and Francis Smith for help preparing the auditory 1112 stimuli, and Tim Griffiths for thoughtful guidance throughout this project. This project was

1113 funded by DC 000242 awarded to B. Gantz, T. Griffiths, and B. McMurray, W81XWH1910637

1114 awarded to I. Choi, DC 008089 awarded to B. McMurray, and NIH T32 5T32DC000040-24

1115 awarded to Bruce Gantz. Jason Geller is now at the Center for Cognitive Science, Rutgers

1116 University. Ann Holmes is now in the Department of Psychological and Brain Sciences, 1117 University of Louisville.

1118 
1123 Figure 10. Number of trials on which a given target consonant was presented with a 1124 corresponding foil consonant.

1125

1126

1127
Target-Foil count for the ITCP

Foil

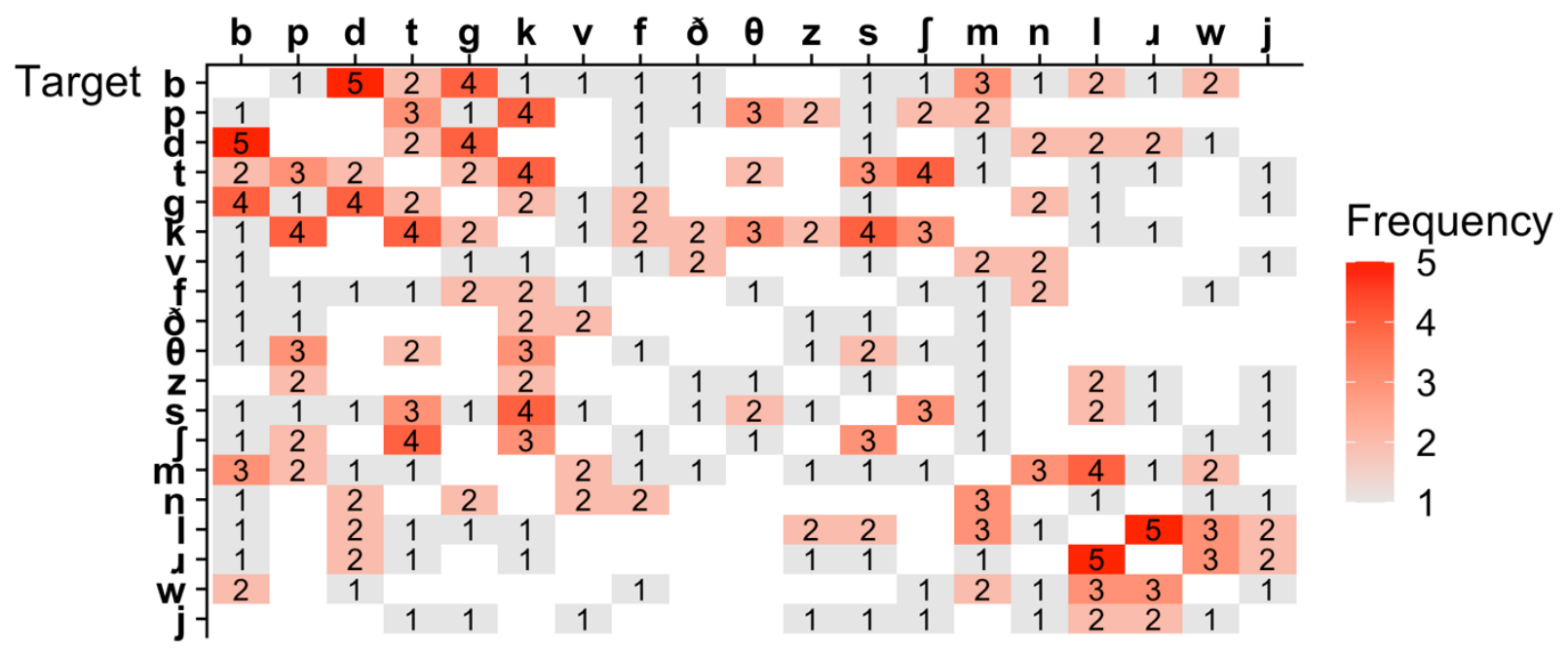

Note. In the validation latch was used as a foil in lieu of thatch for the targets batch and match) so there are some asymmetries (e.g., $b-1=2$, but $1-b=1)$. 
Figure 11. Confusion matrix showing response accuracy given a target phoneme.

1135

\section{Confusion Matrix Session 1 (N=199)}

\begin{tabular}{|c|c|c|c|c|c|c|c|c|c|c|c|c|c|c|c|c|c|c|c|c|}
\hline \multicolumn{21}{|c|}{ Response } \\
\hline & & b & $\mathbf{p}$ & d & $\mathbf{t}$ & $\mathbf{g}$ & $\mathbf{k}$ & v & $f$ & ठ & $\theta$ & $\mathbf{z}$ & $\mathbf{S}$ & $\int$ & $\mathbf{m}$ & $\mathbf{n}$ & 1 & $\mathbf{w}$ & $\mathbf{j}$ & \\
\hline ye & b- & 0.668 & 0.012 & 0.062 & $20.007 c$ & 0.066 & 60.026 & $0.034 C$ & 0.037 & 0.005 & & & 0.014 & 10.006 & 0.0270 & 0.004 & 0.0080 .007 & 0.015 & & \\
\hline & $\mathbf{p - 1}$ & 0.015 & 0.658 & & $0.076 \mathrm{C}$ & 0.005 & 50.125 & & 0.011 & 0.019 & 0.05 & 0.01 & 0.01 & 0.01 & 0.01 & & & & & \\
\hline & $\mathbf{d}$ & 0.05 & & 0.77 & 0.0110 & 0.099 & & & 0.018 & & & & 0.024 & & & 0.009 & 0.0090 .008 & 0.001 & & \\
\hline & $\mathbf{t}-$ & 0.005 & 0.064 & 0.003 & 30.7990 & 0.009 & 90.048 & & 0.006 & & 0.019 & & 0.018 & 30.015 & 0.003 & & 0.0040 .001 & & 0.005 & \\
\hline & g & 0.057 & 0.038 & 30.058 & $80.011 \mathrm{C}$ & 0.758 & 30.02 & $0.004 C$ & 0.024 & & & & 0.004 & & & 0.013 & 30.003 & & 0.011 & \\
\hline & $\mathbf{k}$ & 0.009 & 0.089 & & 0.0420 & 0.016 & 0.729 & $0.006 c$ & 0.023 & 30.007 & 0.039 & 90.006 & 60.019 & 0.009 & & & 0.0030 .005 & & & \\
\hline & $\mathbf{v}$ & 0.04 & & & & 0.058 & 80.008 & $0.672 c$ & 0.013 & 30.108 & & & 0.017 & & 0.0350 & 0.031 & & & 0.018 & \\
\hline & $\mathbf{f}$ & 0.017 & 0.017 & 0.013 & 30.0120 & & 10.017 & 0.027 & 0.745 & & 0.036 & & & 0.046 & 0.010 & 0.023 & & 0.008 & & Proportion \\
\hline & o & 0.045 & 0.014 & & & & 0.039 & 0.246 & & 0.552 & & 0.043 & 30.048 & & 0.013 & & & & & \\
\hline & $\theta-$ & 0.049 & 0.035 & & 0.034 & & 0.028 & & 0.086 & & 0.695 & 50.009 & 90.048 & 30.007 & 0.008 & & & & & 0.15 \\
\hline & $\mathbf{z}$ & & 0.006 & & & & 0.003 & & & 0.012 & 20.001 & 0.947 & 70.006 & & 0.003 & & 0.0060 .009 & & 0.007 & 0.25 \\
\hline & $\mathbf{s}$ & 0.001 & 0.002 & 0.001 & 10.0060 & 0.005 & 0.007 & 0.002 & & 0.006 & 0.007 & 0.005 & 50.918 & 0.034 & 0.001 & & 0.0030 .001 & c & 0.002 & 0.00 \\
\hline & $\int-$ & 0.005 & 0.006 & & 0.016 & & 0.006 & & 0.017 & & 0.002 & & 0.01 & 0.928 & 0.001 & & & 0.002 & 0.006 & \\
\hline & $\mathbf{m}-$ & 0.028 & 0.045 & 0.01 & 0.011 & & & 0.017 & 0.003 & 30.009 & & 0.004 & 40.006 & 0.003 & 30.6960 & 0.072 & 0.0440 .025 & 0.026 & & \\
\hline & $\mathbf{n}$ & 0.01 & & 0.051 & & 0.065 & & 0.063 & 30.036 & & & & & & 0.180 & 0.539 & 0.026 & 0.015 & 0.016 & \\
\hline & $1-$ & 0.006 & & 0.013 & 30.0120 & 0.005 & 50.011 & & & & & 0.022 & 20.014 & & 0.060 & 0.019 & 0.7030 .073 & 0.04 & 0.023 & \\
\hline & $d-$ & 0.013 & & 0.039 & 90.009 & & 0.008 & & & & & 0.005 & 50.01 & & 0.032 & & 0.1350 .652 & 0.079 & 0.017 & \\
\hline & $\mathbf{w}$ & 0.075 & & 0.007 & & & & & 0.016 & & & & & 0.01 & 0.0550 & 0.027 & 0.0860 .087 & 0.621 & 0.016 & \\
\hline & $\mathbf{j}-$ & & & & 0.008 & 0.05 & & 0.014 & & & & 0.017 & 70.005 & 0.005 & & 0.016 & 0.0440 .05 & 0.03 & 0.761 & \\
\hline
\end{tabular}


1139

1140

1141

1142

1143

1144

1145

1146

1147

1148

1149

1150

1151

1152

1153

1154

1155

Figure 12. Easiness Plots for ITCP Items.

Appendix C

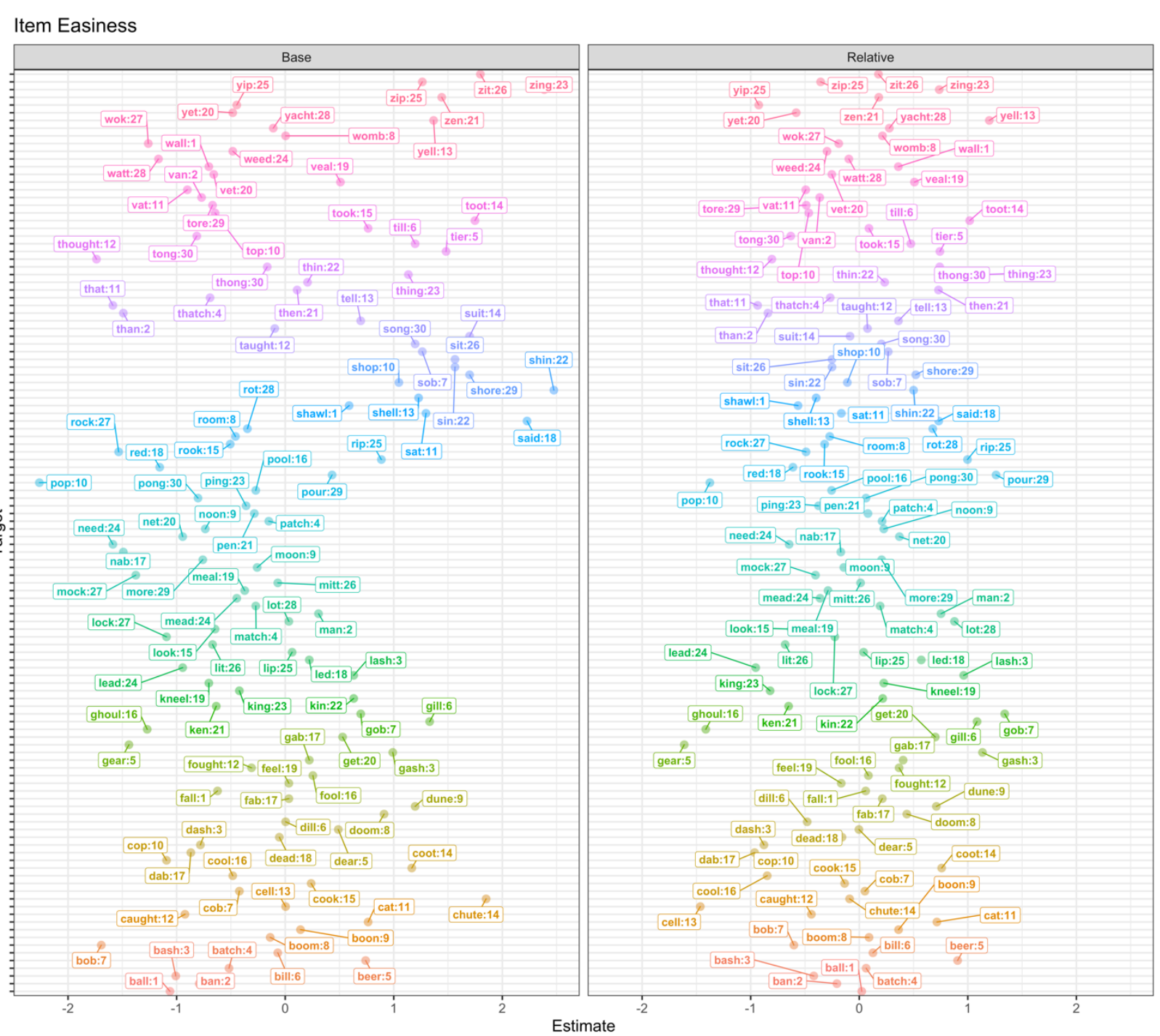

Note. Base model with no factors included (left panel). B. Conditionalized model with all factors included (right panel). Each dot represents an item and the numerical values represents the items corresponding set number. Positive estimates refer to how easy an item is; negative estimates refer to how difficult an item is. 
1156

1157

1158

1159

1160

1161

1162

1163

1164

1165

1166

1167

1168

1169

1170

1171

1172

1173

1174

1175

1176

1177

1178

1179

1180

1181

1182

1183

1184

1185

1186

1187

1188

1189

1190

1191

1192

1193

1194

1195

1196

1197

1198

1199

1200

1201

\section{References}

Allen, M., Poggiali, D., Whitaker, K., Marshall, T. R., \& Kievit, R. A. (2019). Raincloud plots: A multi-platform tool for robust data visualization. Wellcome Open Research, 4. https://doi.org/10.12688/wellcomeopenres.15191.1

Altmann, G. T. M. (1998). Ambiguity in sentence processing. Trends in Cognitive Sciences, 2(4), 146-152). https://doi.org/10.1016/S1364-6613(98)01153-X

Anwyl-Irvine, A. L., Massonnié, J., Flitton, A., Kirkham, N., \& Evershed, J. K. (2020). Gorilla in our midst: An online behavioral experiment builder. Behavior Research Methods, 52(1), 388-407. https://doi.org/10.3758/s13428-019-01237-x

Audacity Team (2012). Audacity. Version 2.0.0. Audio editor and recorder. Available from: http://audacityteam.org/.

Bates, D., Mächler, M., Bolker, B. M., \& Walker, S. C. (2015). Fitting linear mixed-effects models using lme4. Journal of Statistical Software, 67(1), 1-48. https://doi.org/10.18637/jss.v067.i01

Barr, D. J., Levy, R., Scheepers, C., \& Tily, H. J. (2013). Random effects structure for confirmatory hypothesis testing: Keep it maximal. Journal of Memory and Language, 68(3), 255-278. https://doi.org/10.1016/j.jml.2012.11.001

Becker, R., Pefkou, M., Michel, C. M., \& Hervais-Adelman, A. G. (2013). Left temporal alphaband activity reflects single word intelligibility. Frontiers in Systems Neuroscience, 7(DEC). https://doi.org/10.3389/fnsys.2013.00121

Beddor, P. S., \& Krakow, R. A. (1999). Perception of coarticulatory nasalization by speakers of English and Thai: evidence for partial compensation. The Journal of the Acoustical Society of America, 106(5), 2868-2887. https://doi.org/10.1121/1.428111

Bidelman, G.M. \& Howell, M. (2016) Functional changes in inter-and intra-hemispheric cortical processing underlying degraded speech perception. NeuroImage, 124, 581-590.

Black, J. W. (1957). Multiple-choice intelligibility tests. Journal of Speech and Hearing Disorders, 22(2), 213-235. https://doi.org/10.1044/jshd.2202.213

Blarney, P. J., Dowell, R. C., Brown, A. M., Clark, G. M., \& Seligman, P. M. (1987). Vowel and consonant recognition of cochlear implant patients using formant-estimating speech processors. Journal of the Acoustical Society of America, 82(1), 48-57. https://doi.org/10.1121/1.395436

Brysbaert, M., \& New, B. (2009). Moving beyond Kučera and Francis: A critical evaluation of current word frequency norms and the introduction of a new and improved word frequency measure for American English. Behavior Research Methods, 41(4), 977-990. https://doi.org/10.3758/BRM.41.4.977

Chen, Q., \& Mirman, D. (2012). Competition and cooperation among similar representations: Toward a unified account of facilitative and inhibitory effects of lexical neighbors. Psychological Review, 119(2), 417-430. https://doi.org/10.1037/a0027175

Clements, G. (1990). The role of the sonority cycle in core syllabification. In J. Kingston \& M. Beckman (Eds.), Papers in laboratory phonology 1: between the grammar and physics of speech (pp. 283-333). Cambridge: Cambridge University Press.

Clopper, C. G., Pisoni, D. B., \& Tierney, A. T. (2006). Effects of open-set and closed-set task demands on spoken word recognition. Journal of the American Academy of Audiology, 
1202

1203

1204

1205

1206

1207

1208

1209

1210

1211

1212

1213

1214

1215

1216

1217

1218

1219

1220

1221

1222

1223

1224

1225

1226

1227

1228

1229

1230

1231

1232

1233

1234

1235

1236

1237

1238

1239

1240

1241

1242

1243

1244

1245

1246

1247
17(5), 331-349. https://doi.org/10.3766/jaaa.17.5.4

Conrad, C. (1974). Context effects in sentence comprehension: A study of the subjective lexicon. Memory \& Cognition, 2(1-A), 130-138. https://doi.org/10.3758/BF03197504

Coady, J. A., \& Evans, J. L. (2008). Uses and interpretations of non-word repetition tests in children with and without specific language impairments (SLI). International Journal of Language and Communication Disorders, 43(1), 1-40. https://doi.org/10.1080/13682820601116485

Cole, J., Linebaugh, G., Munson, C., \& McMurray, B. (2010). Unmasking the acoustic effects of vowel-to-vowel coarticulation: A statistical modeling approach. Journal of Phonetics, 38(2), 167-184. https://doi.org/10.1016/j.wocn.2009.08.004

Craik, F. I. M., \& Bialystok, E. (2006,). Cognition through the lifespan: Mechanisms of change. Trends in Cognitive Sciences, 10(3), 131-138. https://doi.org/10.1016/j.tics.2006.01.007

Crump, M. J. C., McDonnell, J. V., \& Gureckis, T. M. (2013). Evaluating amazon's

mechanical turk as a tool for experimental behavioral research. PLoS ONE, 8(3), e57410. https://doi.org/10.1371/journal.pone.0057410

Cutler, A., \& Butterfield, S. (1992). Rhythmic cues to speech segmentation: Evidence from juncture misperception. Journal of Memory and Language, 31(2), 218-236. https://doi.org/10.1016/0749-596X(92)90012-M

Dahan, D., \& Tanenhaus, M. K. (2004). Continuous mapping from sound to meaning in spokenlanguage comprehension: Immediate effects of verb-based thematic constraints. Journal of Experimental Psychology: Learning, Memory, and Cognition, 30(2), 498513. https://doi.org/10.1037/0278-7393.30.2.498

Dahan, D., \& Magnuson, J. S. (2006). Spoken-word recognition. In M. J. Traxler \& M. A. Gernsbacher (Eds.), Handbook of Psycholinguistics (pp. 249-283). Amsterdam: Academic Press.

Dawson, C., Aalto, D., Simko, J., \& Vainio, M. (2017). The influence of fundamental frequency on perceived duration in spectrally comparable sounds. PeerJ, 2017(9). https://doi.org/10.7717/peerj.3734

De Boeck, P. (2008). Random Item IRT Models. Psychometrika, 73(4), 533. doi:10.1007/s11336-008-9092-X

Delattre, P. C., Liberman, A. M., \& Cooper, F. S. (1955). Acoustic loci and transitional cues for consonants. The Journal of the Acoustical Society of America, 27(4), 769-773. https://doi.org/10.1121/1.1908024

Donaldson, G. S., \& Kreft, H. A. (2006). Effects of vowel context on the recognition of initial and medial consonants by cochlear implant users. Ear and hearing, 27(6), 658-677. https://doi.org/10.1097/01.aud.0000240543.31567.54

Du, Y., Buchsbaum, B.R., Grady, C.L. \& Alain, C. (2014) Noise differentially impacts phoneme representations in the auditory and speech motor systems. Proceedings of the National Academy of Sciences, 111(19), 7126-7131.

Elliott, L. L., Clifton, L. A. B., \& Servi, D. G. (1983). Word frequency effects for a closed-set word identification task. International Journal of Audiology, 22(3), 229-240. https://doi.org/10.3109/00206098309072787

Foster, J. R., \& Haggard, M. P. (1987). The four alternative auditory feature test (FAAF)linguistic and psychometric properties of the material with normative data in noise. British Journal of Audiology, 21(3), 165-174. https://doi.org/10.3109/03005368709076402

Fox, J., \& Weisberg, S. (2019). An R Companion to Applied Regression, Third Edition. 
Thousand Oaks CA: Sage.

Gamer, M., Lemon, J., Fellows, I., \& Singh, P. (2019). irr: Various coefficients of interrater reliability and agreement. Retrieved from https://CRAN.R-project.org/package=irr

Ganong, W. F. (1980). Phonetic categorization in auditory word perception. Journal of Experimental Psychology: Human Perception and Performance, 6(1), 110-125. https://doi.org/10.1037/0096-1523.6.1.110

Geers, A., \& Brenner, C. (1994). Speech perception results: Audition and lipreading enhancement. Volta Review, 96.

Glosser, G., Kohn, S. E., Friedman, R. B., Sands, L., \& Grugan, P. (1997). Repetition of single words and nonwords in Alzheimer's disease. Cortex, 33(4), 653-666. https://doi.org/10.1016/s0010-9452(08)70723-8

Goldinger, S. D. (1996). Words and voices: episodic traces in spoken word identification and recognition memory. Journal of Experimental Psychology: Learning, Memory, and Cognition, 22(5), 1166.

Gordon, J. K. (2002). Phonological neighborhood effects in aphasic speech errors: Spontaneous and structured contexts. Brain and Language, 82(2), 113-145. https://doi.org/10.1016/S0093-934X(02)00001-9

Grootswagers, T. (n.d.). A primer on running human behavioural experiments online. https://doi.org/10.3758/s13428-020-01395-3

Hickok, G., \& Poeppel, D. (2007). The cortical organization of speech processing. Nature Reviews Neuroscience, 8, 393-402. https://doi.org/10.1038/nrn2113

House, A. S., Williams, C. E., Heker, M. H., and Kryter, K. D. (1965). Articulation-testing methods: Consonantal differentiation with a closed-response set. Journal of the Acoustical Society of America, 37, 158-166.

Hughes, S. E., Hutchings, H. A., Rapport, F. L., McMahon, C. M., \& Boisvert, I. (2018). Social connectedness and perceived listening effort in adult cochlear implant users. Ear and Hearing, 39(5), 922-934. https://doi.org/10.1097/AUD.0000000000000553

Just, M. A., \& Carpenter, P. A. (1992). A capacity theory of comprehension: Individual differences in working memory. Psychological Review, 99(1), 122-149. https://doi.org/10.1037/0033-295X.99.1.122

Killion, M. C., Niquette, P. A., Gudmundsen, G. I., Revit, L. J., \& Banerjee, S. (2004). Development of a quick speech-in-noise test for measuring signal-to-noise ratio loss in normal-hearing and hearing-impaired listeners. The Journal of the Acoustical Society of America, 116(4), 2395-2405. https://doi.org/10.1121/1.1784440

Kim, S., Schwalje, A. T., Liu, A. S., Gander, P. E., McMurray, B., Griffiths, T. D., \& Choi, I. (2021). Pre-and post-target cortical processes predict speech-in-noise performance. Neuroimage, 228, 117699.

Koo, T. K., \& Li, M. Y. (2016). A guideline of selecting and reporting intraclass correlation coefficients for reliability research. Journal of Chiropractic Medicine, 15(2), 155-163. https://doi.org/10.1016/j.jcm.2016.02.012

Lehiste, I., \& Peterson, G. E. (1959). Linguistic considerations in the study of speech intelligibility. The Journal of the Acoustical Society of America, 31(3), 280-286. https://doi.org/10.1121/1.1907713

Luce, P. A. (1986). A computational analysis of uniqueness points in auditory word recognition. Perception \& Psychophysics, 39(3), 155-158. https://doi.org/10.3758/BF03212485 
1293

1294

1295

1296

1297

1298

1299

1300

1301

1302

1303

1304

1305

1306

1307

1308

1309

1310

1311

1312

1313

1314

1315

1316

1317

1318

1319

1320

1321

1322

1323

1324

1325

1326

1327

1328

1329

1330

1331

1332

1333

1334

1335

1336

1337

1338
Lenth, R. (2020).Emmeans: Estimated marginalmeans, aka least-squares means. https://github.com/rvlenth/emmeans

Luce, P. A., \& Pisoni, D. B. (1998). Recognizing spoken words: The neighborhood activation model. Ear and Hearing, 19(1), 1-36. https://doi.org/10.1097/00003446-199802000-00001

Mann, V. A., \& Repp, B. H. (1980). Influence of vocalic context on perception of the [J]-[s] distinction. Perception \& Psychophysics, 28(3), 213-228. https://doi.org/10.3758/BF03204377

Marian, V., Bartolotti, J., Chabal, S., \& Shook, A. (2012). CLEARPOND: Cross-linguistic easyaccess resource for phonological and orthographic neighborhood densities. PLoS ONE, 7(8), e43230. https://doi.org/10.1371/journal.pone.0043230

Martin, F. N., Champlin, C. A., \& Perez, D. D. (2000). The question of phonetic balance in word recognition testing. Journal of the American Academy of Audiology, 11(9), 509-513.

Mason, W., \& Suri, S. (2012). Conducting behavioral research on Amazon's Mechanical Turk. Behavior Research Methods, 44(1), 1-23. https://doi.org/10.3758/s13428-011-0124-6

Matheson, G. J. (2019). We need to talk about reliability: Making better use of test-retest studies for study design and interpretation. PeerJ, 2019(5). https://doi.org/10.7717/peerj.6918

McClelland, J. L., \& Elman, J. L. (1986). The TRACE model of speech perception. Cognitive Psychology, 18(1), 1-86. https://doi.org/10.1016/0010-0285(86)90015-0

McMurray, B., \& Jongman, A. (2011). What Information Is Necessary for Speech Categorization? Harnessing Variability in the Speech Signal by Integrating Cues Computed Relative to Expectations. Psychological Review, 118(2), 219-246. https://doi.org/10.1037/a0022325

Middleton, E. L., \& Schwartz, M. F. (2010). Density pervades: An analysis of phonological neighbourhood density effects in aphasic speakers with different types of naming impairment. Cognitive Neuropsychology, 27(5), 401-427.

https://doi.org/10.1080/02643294.2011.570325

Miller, G. A., \& Nicely, P. A. (1955). An analysis of perceptual confusions among some English consonants. Journal of the Acoustical Society of America, 27, 338352. https://doi.org/10.1121/1.1907526

Mueller, H. (2001). Speech audiometry and hearing aid fittings: Going steady or casual acquaintances? Hearing Journal, 54(10), 19-29. https://doi.org/10.1097/01.hj.0000294535.51460.0c

Munson, B., Johnson, J. M., \& Edwards, J. (2012). The role of experience in the perception of phonetic detail in children's speech: A comparison between speech-language pathologists and clinically untrained listeners. American Journal of Speech-Language Pathology, 21(2), 124-139. https://doi.org/10.1044/1058-0360(2011/11-0009)

Nakajima, Y., Matsuda, M., Ueda, K., \& Remijn, G. B. (2018). Temporal Resolution Needed for Auditory Communication: Measurement with Mosaic Speech. Frontiers in Human Neuroscience, 12, 149

Newman, R. S., Clouse, S. A., \& Burnham, J. L. (2001). The perceptual consequences of withintalker variability in fricative production. The Journal of the Acoustical Society of America, 109(3), 1181-1196. https://doi.org/10.1121/1.1348009

Nilsson, M., Soli, S. D., \& Sullivan, J. A. (1994). Development of the hearing in noise test for the measurement of speech reception thresholds in quiet and in noise. Journal of the Acoustical Society of America, 95(2), 1085-1099. https://doi.org/10.1121/1.408469

Novick, J. M., Trueswell, J. C., \& Thompson-Schill, S. L. (2005). Cognitive control and parsing: 
reexamining the role of Broca's area in sentence comprehension. Cognitive, affective \& behavioral neuroscience, 5(3), 263-281. https://doi.org/10.3758/cabn.5.3.263

Nygaard, L. C., Sommers, M. S., \& Pisoni, D. B. (1994). Speech perception as a talkercontingent process. Psychological Science, 5(1), 42-46. https://doi.org/10.1111/j.14679280.1994.tb00612.x

Owens, E., \& Schubert, E. D. (1977). Development of the California consonant test. Journal of Speech and Hearing Research, 20(3), 463-472. https://doi.org/10.1044/jshr.2003.46

Paolacci, G., Chandler, J., Ipeirotis, P. G., \& Stern, L. N. (2010). Running experiments on Amazon Mechanical Turk. Judgment and Decision Making, 5(5), 411-419.

Patil, I. (2021). Visualizations with statistical details: The 'ggstatsplot' approach. Journal of Open Source Software, 6(61), 3167, doi:10.21105/joss.03167

Peelle, J. E., \& Wingfield, A. (2016). The Neural consequences of age-related hearing loss. Trends in Neurosciences, 39(7), 486-497. https://doi.org/10.1016/j.tins.2016.05.001

Pernet, C. R., Wilcox, R., \& Rousselet, G. A. (2013). Robust correlation analyses: False positive and power validation using a new open source Matlab toolbox. Frontiers in Psychology, 3(JAN), 606. https://doi.org/10.3389/fpsyg.2012.00606

Pichora-Fuller, M. K., Kramer, S. E., Eckert, M. A., Edwards, B., Hornsby, B. W. Y., Humes, L. E., ... Wingfield, A. (2016). Hearing impairment and cognitive energy. Ear and Hearing, 37, 5S-27S. https://doi.org/10.1097/AUD.0000000000000312

Pinet, S., Ziegler, J. C., \& Alario, F. X. (2016). Typing is writing: Linguistic properties modulate typing execution. Psychonomic Bulletin \& Review, 23(6), 1898-1906

Redmond, S. M., Ash, A. C., Christopulos, T. T., \& Pfaff, T. (2019). Diagnostic accuracy of sentence recall and past tense measures for identifying children's language impairments. Journal of Speech, Language, and Hearing Research, 62(7), 2438-2454. doi: 10.1044/2019_JSLHR-L-18-0388

Remez, R., Rubin, P., Pisoni, D., \& Carrell, T. (1981). Speech perception without traditional speech cues. Science, 212(4497), 947-949. https://doi.org/10.1126/science.7233191

Rigler, H., Farris-Trimble, A., Greiner, L., Walker, J., Tomblin, J. B., \& McMurray, B. (2015). The slow developmental time course of real-time spoken word recognition. Developmental Psychology, 51(12), 1690-1703. https://doi.org/10.1037/dev0000044

Rogalsky, C., Poppa, T., Chen, K. H., Anderson, S. W., Damasio, H., Love, T., \& Hickok, G. (2015). Speech repetition as a window on the neurobiology of auditory-motor integration for speech: A voxel-based lesion symptom mapping study. Neuropsychologia, 71, 18-27. https://doi.org/10.1016/j.neuropsychologia.2015.03.012

Rönnberg, J., Rudner, M., Foo, C., \& Lunner, T. (2008). Cognition counts: A working memory system for ease of language understanding (ELU). International Journal of Audiology, 47(sup2), S99-S105. https://doi.org/10.1080/14992020802301167

Sadagopan, N., \& Smith, A. (2008). Developmental changes in the effects of utterance length and complexity on speech movement variability. Journal of Speech, Language, and Hearing Research, 51(5), 1138-1151. https://doi.org/10.1044/1092-4388(2008/06-0222)

Salverda, A. P., Dahan, D., \& McQueen, J. M. (2003). The role of prosodic boundaries in the resolution of lexical embedding in speech comprehension. Cognition, 90(1), 51-89. https://doi.org/10.1016/S0010-0277(03)00139-2

Sarampalis, A., Kalluri, S., Edwards, B., \& Hafter, E. (2009). Objective measures of listening 
1383

1384

1385

1386

1387

1388

1389

1390

1391

1392

1393

1394

1395

1396

1397

1398

1399

1400

1401

1402

1403

1404

1405

1406

1407

1408

1409

1410

1411

1412

1413

1414

1415

1416

1417

1418

1419

1420

1421

1422

1423

1424

1425

1426

1427

1428 effort: Effects of background noise and noise reduction. Journal of Speech, Language, and Hearing Research, 52(5), 1230-1240. https://doi.org/10.1044/1092-4388(2009/08-0111)

Seedorf, M., Oleson, J., McMurray, B. (2020). Maybe maximal: Good enough mixed models optimize power while controlling Type I error. Psyarxiv. https://psyarxiv.com/xmhfr/

Singh, S., \& Black, J. W. (1966). Study of twenty-six intervocalic consonants as spoken and recognized for four language groups. Journal of the Acoustical Society of America, 39(2), 372-387. https://doi.org/10.1121/1.1909899

Shinn-Cunningham B (2019). Brain mechanisms of auditory scene analysis., in M. Gazzaniga, G. Mangun, G., and D. Poeppel (eds.) The Cognitive Neurosciences VI (pp 159-166). Cambridge, MA: MIT Press.

Slote, J., \& Strand, J. F. (2016). Conducting spoken word recognition research online: Validation and a new timing method. Behavior Research Methods, 48(2), 553-566. https://doi.org/10.3758/s13428-015-0599-7

Sommers, M. S., Kirk, K. I., \& Pisoni, D. B. (1997). Some considerations in evaluating spoken word recognition by normal-hearing, noise-masked normal-hearing, and cochlear implant listeners. I: The effects of response format. Ear and Hearing, 18(2), 89-99. https://doi.org/10.1097/00003446-199704000-00001

Spahr, A. J., Dorman, M. F., Litvak, L. M., Van Wie, S., Gifford, R. H., Loizou, P. C., ... Cook, S. (2012). Development and validation of the AzBio sentence lists. Ear and Hearing, 33(1), 112-117. https://doi.org/10.1097/AUD.0b013e31822c2549

Tanenhaus, M.K. \& Trueswell, J.C. (1995) Sentence comprehension. In P.D. Eimas and J.L. Miller (Eds.) Handbook in perception and Cognition. Speech, language and communication. (p. 217-262), New York, NY: Academic Press.

Taylor, B. (2003). Speech-in-noise tests: How and why to include them in your basic test battery. Hearing Journal, 56(1), 40-46. https://doi.org/10.1097/01.HJ.0000293000.76300.ff

Torretta, G. (1995). The "easy-hard" word multi-talker speech database: an initial report. Research on Spoken Language Processing, Progress Report 20. Bloomington: Speech Research Laboratory, Department of Psychology, Indiana University.

Tyler, R. S., Lowder, M. W., Otto, S. R., Preece, J. P., Gantz, B. J., \& McCabe, B. F. (1984). Initial Iowa results with the multichannel cochlear implant from Melbourne. Journal of Speech and Hearing Research, 27(4), 596-604. https://doi.org/10.1044/jshr.2704.596

Voiers, W. D. (1977). Diagnostic evaluation of speech intelligibility. In M. E. Hawley (Ed.), Speech intelligibility and speaker recognition (pp. 374-387). Stroudsburg, PA: Dowden, Hutchinson, \& Ross.

Voiers, W. D. (1983). Evaluating processed speech using the Diagnostic Rhyme Test. Speech Technology, 3, 30-39.

Walden, B. E., Holum-Hardegen, L. L., Crowley, J. M., Schwartz, D. M., \& Williams, D. L. (1983). Test of the assumptions underlying comparative hearing aid evaluations. Journal of Speech and Hearing Disorders, 48(3), 264-273. https://doi.org/10.1044/jshd.4803.264

Wang, M. D., \& Bilger, R. C. (1973). Consonant confusions in noise: A study of perceptual features. Journal of the Acoustical Society of America, 54(5), 1248-1266. https://doi.org/10.1121/1.1914417

Wilcox, R. R. (1994). The percentage bend correlation coefficient. Psychometrika, 59(4), 601616. https://doi.org/10.1007/BF02294395

Wilson, R. H. (2003). Development of a speech-in-multitalker-babble paradigm to assess wordrecognition performance. Journal of the American Academy of Audiology, 14(9), 453-470. 
1429 Woods, K. J. P., Siegel, M. H., Traer, J., \& McDermott, J. H. (2017). Headphone screening to

1430

1431

1432

1433

1434

1435

1436

1437

1438

1439

1440

1441

1442

1443

1444

1445

1446

1447

1448

1449

1450

1451

1452

1453

1454 facilitate web-based auditory experiments. Attention, Perception, and Psychophysics, 79(7), 2064-2072. https://doi.org/10.3758/s13414-017-1361-2

Wong, P.Cs.M., Uppunda, A.K., Parrish, T.B. \& Dhar, S. (2008) Cortical mechanisms of speech perception in noise. Journal of Speech, Language, and Hearing Research, 51(4), 10261041.

(1)


Table 1. Demographic Variables $(\mathrm{N}=97)$

\begin{tabular}{ll} 
Factor & M / N (range / \%) \\
\hline Age & 27 years $(23,35)$ \\
Gender & $48(49 \%)$ \\
Female & $48(49 \%)$ \\
Male & $1(1.0 \%)$ \\
Other & \\
Race & $9(22 \%)$ \\
Asian & $11(27 \%)$ \\
Black or African & \\
American & $21(51 \%)$ \\
White & 56 \\
Unknown &
\end{tabular}

1456

Table 2. Model Summary for Full Model

1458

\begin{tabular}{lccc}
\hline Variable & $\chi^{2}$ & DF & $\boldsymbol{p}$ \\
\hline isStop & 0.186 & 1 & 0.666 \\
isFric & 0.067 & 1 & 0.796 \\
Voicing & 0.610 & 1 & 0.435 \\
labialstop & 10.235 & 1 & 0.001 \\
velarstop & 2.712 & 1 & 0.100 \\
labiodentalfric & 35.920 & 1 & $<.001$ \\
interdentalfric & 41.29 & 1 & $<.001$ \\
Alvelorfric & 0.224 & 1 & 0.635 \\
Sonorant1 & 0.005 & 1 & 0.946 \\
Sonorant2 & 4.469 & 1 & 0.035 \\
Sonorant3 & 0.228 & 1 & 0.633 \\
Sonorant4 & 0.010 & 1 & 0.917 \\
Sonorant5 & 1.69 & 1 & 0.193 \\
Talker & 106.73 & 3 & $<.001$ \\
Vowel & 33.78 & 3 & $<.001$ \\
NumVoicingFoils & 0.103 & 1 & 0.749 \\
NumPlaceFoils & 2.60 & 1 & 0.107 \\
NumMannerFoils & 1.06 & 1 & 0.303 \\
\hline
\end{tabular}


1462

1463

1464

1465

1466

1467

1468

1469

1470

1471

1472

1473

1474

1475

1476

1477

1478

1479

1480

1481

1482

1483

1484

1485

1486

1487

1488

1489

1490

1491

1492

1493

1494

1495

1496

1497

1498

1499

1500

1501

1502

1503

1504

1505

1506

1507

1508

1509

1510

1511
Figure 1. (Color online). Distribution of consonants tested in two common speech tests and the ITCP (A. CNC; B. CCT; C. ITCP). Consonants are color coded by manner of articulation, and the width of each arc corresponds to the number of trials.

Figure 2. (Color online). Heat map showing the number of trials that test a specific minimal pair contrast for each phoneme. For example, a $/ \mathrm{b} /$ trial testing place would use either a $/ \mathrm{g} /$ or $/ \mathrm{d} / \mathrm{as}$ a foil; $\mathrm{a} / \mathrm{z} / \mathrm{trial}$ testing voicing would have $/ \mathrm{s} /$ for a foil, and a $/ \mathrm{z} /$ trial testing manner would have $/ \mathrm{n} /, / \mathrm{d} /, / 1 /$ and $/ \mathrm{x} /$ for foils. Note that some contrasts (indicated in white with red boxes) are not possible in word-intial position in English (sonorants cannot contrast voicing, and some places of articulation are found only in fricatives) or were so rare that no minimal pairs could be found. Also note that we did not attempt to collapse neighboring places of articulation that are commonly treated similarly (e.g., / $/$ / velar] and $/ \mathrm{S} /$ [postalveolar]) are treated as a 2-feature contrast [place + manner]); thus many two features changes are actually quite close in acoustic or articulatory space.

Figure 3. (Color online). Distribution of vowels by type in two common speech tests and the ITCP (A. CNC; B. CCT; C. ITCP). Vowels are color coded by manner of articulation, and the width of each arc corresponds to the number of trials.

Figure 4. (Color online). Consonant by vowel-type cooccurrence counts for the 120 items in the ITCP. Color intensity represents magnitude with lighter colors representing a fewer number of trials testing a particular contrast within a phoneme and darker colors representing a greater number of trials testing a particular contrast within a phoneme.

Figure 5. (Color online). Trial structure of the ITCP.

Figure 6. Raincloud plots (Allen et al., 2019) depicting results for Session 1 as a function of place of articulation (separate plots) within each manner of articulation (separate panels). Shown are byparticipant accuracies (dots), medians and quartiles (box plots), grand means (red dots), and distributions of accuracy scores (half violin kernel density plots).

Figure 7. (Color online). Raincloud plots (Allen et al., 2019) depicting proportion correct during Session 1 as a function of Talker. Shown are by-participant accuracies (dots), medians and quartiles (box plots), grand means (red dots), and distributions of accuracy scores (half violin kernel density plots).

Figure 8. (Color online). Raincloud plots depicting proportion correct during Session 1 as a function of vowel quadrant. Shown are by-participant accuracies (dots), medians and quartiles (box plots), grand means (red dots), and distributions of accuracy scores (half violin kernel density plots).

Figure 9. (Color online). A) Scatter plot of proportion correct between Session 1 and Session 2 of the ITCP along with distribution of the data (histograms) B) Scatter plot of proportion correct between Session 1 of the ITCP and AzBio along with distribution of the data (histograms). C) Scatter plot of proportion correct between Session 1 of the ITCP and CNC along with distribution of the data (histograms). D) Scatter plot of proportion correct between AzBio and CNC along with distribution of the data (histograms Plots were created with ggstatsplot (Patil, 2021).

Figure 10 (color online). Number of trials on which a given target consonant was presented with a corresponding foil consonant.

Figure 11 (color online). Confusion matrix showing response accuracy given a target phoneme. 
1512 Figure 12 (color online). Easiness plots for ITCP Items.

1513

1514

1515 